\title{
Le Codex de Bèze : base indispensable pour une édition de l'Évangile de Marc
}

\author{
[Bezae's Codex: an indispensable text-base for an edition of Mark's \\ Gospel] \\ Josep RIUS-CAMPS \\ Facultat de Teologia de Catalunya (Barcelona) \\ riuscamps@yahoo.es
}

Resumen: Inventario de los errores de ortografía detectados en el Evangelio de Marcos así como de la variada utilización del nombre de Jesús o del pronombre por el Codex Bezae y el Codex Vaticanus. El fenómeno de las secuencias desdobladas. Cuestiones planteadas por sus discípulos en un lugar apartado de la muchedumbre sobre los temas ya tratados con anterioridad. Formulación de una hipótesis que ofrezca una explicación plausible de dichos fenómenos a partir del proceso redaccional del Evangelio de Marcos.

Abstract: In this article we give an inventory of the spelling mistakes in the Gospel of Mark, together with an analysis of Jesus' name references or the third person pronoun, including the variant readings of Codex Bezae and Codex Vaticanus. The phenomenon of parallel sequences. The questions of the disciples, apart from the crowd, on themes previously dealt with. A hypothesis for explaining such a phenomena from the redactional process of the Gospel of Mark is also offered.

Palabras Clave: Griego. Evangelio de Marcos. Codex Bezae Cantabrigiensis. Errores.

Key Words: Greek. Gospel of Mark. Codex Bezae Cantabrigiensis. Mistakes.

\section{Introduction}

La thèse principale de l'édition de l'Evangile de Marc dont je prépare la publication (Estella, Navarra: Verbo Divino, 2008) est que le texte grec du Codex de Bèze (D05) est une base indispensable pour comprendre le processus de rédaction de 1 'Évangile de Marc, entrepris par l'auteur même. En raison de sa situation unique parmi les témoins grecs de Marc, je soutiens qu'il est nécessaire de publier le texte, une fois corrigées les erreurs d'orthographe et autres fautes de copiste de ce type, tel qu'il se trouve dans le manuscrit. Dans cette édition je présenterai, dans une colonne face au texte du Codex de Bèze, 
le texte du Codex Vaticanus (B03), représentant principal du texte alexandrin qui est repris presque entièrement dans l'édition critique de Nestle-Aland, Novum Testamentum Graece $\left({ }^{27} \mathrm{NTG}\right)$; l'édition plus restreinte The Greek New Testament $\left({ }^{4} \mathrm{GNT}\right)$ partage le même texte de base. Ainsi, on aura une édition parallèle qui permettra au lecteur de saisir facilement la nature et la quantité des variantes entre le texte habituellement lu et un autre que je juge plus ancien en raison des traces de l'œuvre qu'il laisse voir de son auteur. Le texte grec sera accompagné par une introduction qui exposera en détail l'analyse du texte et de notes qui commenteront les variantes.

Dans la présente étude, suite à cette brève introduction je commenterai les fautes de copie de D05 qui seront corrigées dans l'édition publiée. Je passerai ensuite à une analyse des phénomènes qui m'ont amené à considérer le texte du Codex de Bèze

La grande majorité des exégètes qui font des recherches sur l'Évangile de Marc prennent comme point de départ le texte grec des éditions courantes. C'est un texte éclectique basé essentiellement sur le Codex Vaticanus appuyé souvent par le Codex Sinaiticus (\$01). Il s'agit bien sûr d'une editio minor, mais le fait est qu'elle est devenue, grâce au tirage de presque trois cents mille exemplaires, une sorte de textus communiter acceptus. D'après l'expérience que nous avons fait, Jenny Read-Heimerdinger et moi, d'abord séparément ${ }^{1}$ et maintenant en étroite collaboration, sur le texte des Actes des Apôtres en analysant de très près le Codex de Bèze (grec et latin) et en le comparant à $\aleph 01$ et $\mathrm{B} 03,{ }^{2}$ nous sommes arrivés à la conviction que le Codex de Bèze est d'une cohérence plus marquée - par exemple, dans l'onomastique et l'usage des noms doubles, dans l'utilisation des formes lexicales apparemment synonymes

1 Jenny ReAd-Heimerdinger, The Bezan Text of Acts. A Contribution of Discourse Analysis to Textual Criticism, London-New York : Sheffield Academic Press, 2002.- Josep RIUS-CAMPS, El camino de Pablo a la misión de los paganos. Comentario lingüistico y exegético a Hch 1328, Madrid : Cristiandad, 1984 ; De Jerusalén a Antioquía. Comentario lingüístico y exegético a Hch 1-12, Córdoba : El Almendro 1989 ; Comentari als Fets dels Apòstols. Vol. 1. «Jerusalem» : Configuració de l'església judeocreient (Ac 1,1-5,42), Barcelona : Facultat de Teologia de Catalunya-Editorial Herder, 1991 ; Vol. 2. "Judea i Samaria»: Gènesi de l'església cristiana a Antioquia, Barcelona : Facultat de Teologia de Catalunya-Editorial Herder, 1993 ; Vol. 3. "Fins als confins de la terra» : Primera i segona fases de la missió al paganisme (Ac 13,1-18,23)», Barcelona : Facultat de Teologia de Catalunya-Editorial Herder, 1995 ; Vol. 4. D’Efes a Roma, amb marrada a Jerusalem : Tercera i quarta fases de la missió al paganisme (Ac 18,24-28,31), Barcelona : Facultat de Teologia de Catalunya, 2000.

2 Josep Rius-CAMPS - Jenny READ-HeIMERdinger, The Message of Acts in Codex Bezae. A Comparison with the Alexandrian Tradition. Vol. 1. Acts 1.1-5.42 : Jerusalem, London-New York : T\&T Clark International, 2004 ; Vol. 2. Acts 6.1-12.25 : From Judaea and Samaria to the Church in Antioch, New York-London: T\&T Clark, 2006; Vol. 3. Acts 13.1-18.23 The Ends of the Earth. First and Second Phases of the Mission to the Gentiles, London-New York : T\&T Clark, 2007. 
mais en réalité bien distinctes, dans l'emploi des particules, pour ne citer que quelques exemples.

Muni de ces découvertes, j'avais entrepris, il y a déjà dix ans, l'analyse de toutes les variantes du Codex de Bèze dans l'Évangile de Marc collationnées avec les leçons fournies par $\$ 01$ et B03. À présent, j'ai publié dans la Revista Catalana de Teologia dix-sept notes critiques qui couvrent presque deux tiers de l'évangile, c'est-à-dire Mc 1,1-11,26, et j'ai compté $1089 \mathrm{vll}$, desquelles 549 vll (soit la moitié) n'ont pas été enregistrées dans l'édition critique de Nestle-Aland. ${ }^{3}$ En faisant une extrapolation, le nombre de vll prévisibles pourrait atteindre à peu près 1650 .

Grâce à cet examen attentif du texte de Marc suivant la récension conservée par le Codex de Bèze, je me suis rendu compte qu'il y avait une fluctuation considérable en ce qui concerne l'usage du nom de Jésus non seulement à l'intérieur du Codex de Bèze mais encore par rapport au Codex Vaticanus et qu'il était possible de formuler une hypothèse qui permettrait de distinguer clairement trois niveaux rédactionnels dans l'évangile en son état actuel. Avant de développer en plus de détail mon hypothèse, il m'est indispensable de faire deux remarques d'ordre préliminaire.

\section{Fixation du texte grec}

La première remarque concerne le choix du texte grec à partir duquel il m'a été possible de formuler mon hypothèse. Celle-ci fonctionne bien, en effet, uniquement si l'on part de la forme du texte conservée par le Codex de Bèze. Lorsque j'ai voulu la contrôler à partir d'autres états du texte, par example ceux du Codex Vaticanus ou du Codex Sinaiticus, j'ai dû constater que le point de départ de mon hypothèse n'aurait pas mené à des résultats concluants.

Pour la fixation du texte grec du Codex de Bèze j'ai tenu compte des éditions modernes, mais surtout de l'édition diplomatique du Codex de Bèze faite par F.H. Scrivener. ${ }^{4}$ Il s'agit d'un codex pre-récensionnel bilingue dont la

3 « Les variants de la recensió occidental de l'Evangeli de Marc» I, RCatT 22 (1997) 163-177; II, ibid., 409-419 ; III, RCatT 23 (1998) 195-205 ; IV, ibid., 401-419 ; V, RCatT 24 (1999) 215-230 ; VI-VII, ibid., 419-459 ; VIII, RCatT 26 (2001) 169-186 ; IX, ibid., 365-383 ; X, RCatT 27 (2002) 185-202 ; XI, ibid., 451-464 ; XII, RCatT 28 (2003) 197-212 ; XIII, ibid., 471-488 ; XIV, RCatT 29 (2004) 157-188 ; XV, ibid., 455-475 ; XVI, RCatT 31 (2006) 193237 ; XVII, RCatT 32 (2007) 207-228.

4 Frederick H. SCRIVENER (éd.), BEZAE CODEX CANTABRIGIENSIS, being an exact copy, in ordinary type, of the celebrated uncial greco-latin manuscript of the four Gospels and Acts of the Apostles, written early in the sixth century and presented to the University of Cambridge by Theodore Beza, A. D. 1581. Edited with a critical introduction, annotations and facsimiles, Cambridge : Deighton, Bell and Co., 1864 ; Pittsburgh, Pennsylvania : The Pickwick Press, 1878 [réimpression]). Il existe une édition plus récente faite au Vatican par A. AMMASSARI (Bezae Codex Cantabrigiensis, Città del Vaticano: Libreria Editrice Vaticana, 1996), mais qui 
colonne grecque a échappé presque toujours à la contamination du texte byzantin, devenu dominant dans les églises de la Méditerranée. Ce phénomène était sans doute dû, en partie au moins, à son isolement en Gaule où l'on parlait latin. Dans la colonne latine, au contraire, on peut identifier de fréquentes harmonisations avec les autres versions vielles latines de l'entourage.

On peut aisément remonter bien des leçons uniques du Codex de Bèze au II $^{\mathrm{e}}$ siècle, suivant le témoignage des anciennes versions latines (it), syriaques (sy ${ }^{\text {s.pal.hmg }}$ ) et coptes (co ${ }^{\text {mae }}$ ) qui en sont les meilleurs garants, ainsi que des pères grecs et latins, tels Irénée et Tertullien.

Cependant, la page grecque du Codex de Bèze contient beacoup d'erreurs orthographiques dûes à la prononciation historique de cette langue ${ }^{5}$ et d'autres erreurs causées par le travail des différents scribes qui ont participé à la transmission du texte. Après sa confection vers la fin du IV ${ }^{\mathrm{e}}$ siècle, le Codex de Bèze a été corrigé successivement par plusieurs correcteurs de manière nonsystématique. Vous trouverez ci-dessous toutes les erreurs possibles que j'ai pu identifier dans l'Évangile de Marc d'après le texte conservé par le Codex de Bèze.

Dans une première table (Table 1) on trouvera, pour l'Évangile de Marc selon le Codex de Bèze, les divers types d'erreurs dûes à la prononciation historique des voyelles grecques. Ángel Urbán a déjà dressé l'inventaire et la classification de tous les échanges vocaliques. ${ }^{6}$ Ainsi j'énumérerai seulement les fréquences statistiques dans la table ci-jointe.

Table 1

Échanges vocaliques dans Mc D05

\begin{tabular}{|l|l|c|c|}
\hline Son de la voewlle & Type d'erreur & Nombre d'erreurs & Total \\
\hline Son E & E au lieu de AI & 39 & \\
\hline & AI au lieu de E & 75 & 122 \\
\hline & H au lieu de E & 8 & \\
\hline Son I & I au lieu de EI & 50 & \\
\hline & E au lieu de H & 4 & \\
\hline & EI au lieu de I & 235 & \\
\hline & H au lieu de I & 2 & \\
\hline & Y au lieu de I & 2 & \\
\hline
\end{tabular}

présente de remarquables déficiences surtout en ce qui concerne la page latine (le texte grec n'est qu'une réproduction photomécanique de l'édition fac-similée de Scrivener).

5 Voir le chapîre IV. « The Historical Pronunciation of Greek » de Chrys. C. CARAGOUNIS, The Development of Greek and the New Testament. Morphology, Syntax, Phonology and Textual Transmission, Grand Rapids, Michigan : Baker Academic, 2006, pp. 350-396.

6 «Bezae Codex Cantabrigiensis (D) : intercambios vocálicos en el texto de Marcos», Collectanea Christiana Orientalia 4 (2007) 245-268. 


\begin{tabular}{|l|l|c|c|}
\hline & Y au lieu de OI & 3 & \\
\hline & OY au lieu de Y & 2 & 298 \\
\hline Son $O$ & O au lieu de $\Omega$ & 3 & \\
\hline & OY pour $\Omega$ & 3 & 6 \\
\hline Grand total & & & 426 \\
\hline
\end{tabular}

Le nombre total d'erreurs de pronunciation identifiées en D05 s'élève à 426. L'erreur la plus fréquente est celle du son I (itacisme), notamment la confusion de I au lieu de EI $(50 \times)$ et viceversa EI au lieu de I $(235 \times)$, suivie de celle du son $\mathrm{E}$ (etacisme), notamment la confusion de $\mathrm{E}$ au lieu de AI $(39 \times)$ et viceversa, AI au lieu de $\mathrm{E}(75 \times)$.

Dans une deuxième table (Table 2) j'ai fait l'inventaire et la classification de toutes les autres erreurs que j'ai pu identifier dans D05, en signalant s'il y a des corrections et en évaluant si telle erreur possible est ou non une erreur réelle et si telle ou telle correction est justifiée ou bien si elle provient d'une harmonisation avec le texte devenu majoritaire.

Dans la première colonne je donne la référence (Réf.) de la variante ; dans la deuxième, le numéro du folio et de la ligne correspondante (Fol./l.); dans la troisième, la Leçon originale de D05 (p.m.) ; dans la quatrième, les Corrections qui figurent dans les Adnotationes editoris ${ }^{7}$ de l'éditeur Scrivener ou des corrections propres ; dans la cinquième sont annotés (Ann.) les sigles des correcteurs (s.m., $\mathrm{D}^{\mathrm{A}}, \mathrm{D}^{\mathrm{B}}, \mathrm{D}^{\mathrm{C}}$, etc.), des corrections propres (corr.), des conjectures (cj., une conjecture propre), des annotations de Scrivener (Scr.) ou des leçons que je considère tout à fait bonnes (b.l.) ; dans la sixième figurent les Harmonisations (Harm.) avec B03 que j'ai pu déceler ; dans la septième je donne la Bonne leçon ; finalement, dans la huitième colonne $(O r$. ) je signale avec un astérisque quand celle-la que j'ai considérée comme la bonne leçon coïncide à peu pres avec la leçon originale de D05 et non pas avec la leçon proposée par le correcteur ou celle attestée par B03, ces dernières leçons n'ayant pas été enregistrées en général comme variantes dans l'édition vingt-septième de Nestle-Aland (sigle : B $v l$ n.e.).

Table 2

Table des erreurs possibles dans Mc D05

\begin{tabular}{|c|c|c|c|c|c|c|}
\hline Réf. & Fol. / l. & Leçon originale & Correction & Ann.. & Harm. & Bonne leçon \\
\hline 1,5 & $\begin{array}{c}285 b / 13 \\
,\end{array}$ & $\begin{array}{l}\text { EN IOP } \triangle A N H \\
\text { KAME } \triangle O Y\end{array}$ & $\begin{array}{l}\epsilon \nu \tau \omega \text { io } \rho \delta \alpha \nu \eta \\
\kappa \alpha \mu \eta \lambda \text { ou }\end{array}$ & $\begin{array}{l}D^{C} \\
D^{C}\end{array}$ & B $v l$ n.e. & 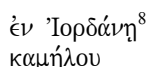 \\
\hline
\end{tabular}

SCRIVENER, BEZAE CODEX CANTABRIGIENSIS, 429ss.

Cf. B-D-R, §261, n. 9 : « Josephus lässt im Bell.Jud. gewöhnlich den Artikel aus. » Pour Marc c'est la première mention. Voir « Les variants » $I, n^{\circ} 6$. 


\begin{tabular}{|c|c|c|c|c|c|c|}
\hline 1,9 & $" / 25$ & 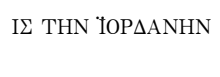 & 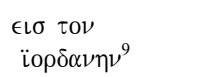 & $D^{A . D}$ & & 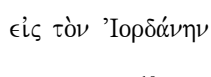 \\
\hline 1,10 & $" / 28$ & KATABAIN $\Omega N$ & $\kappa \alpha \tau \alpha \beta \alpha \iota \nu 0 \nu$ & $\mathrm{D}^{\mathrm{D}}$ & B $v l$ n.e. & $\kappa \alpha \tau \alpha \beta \alpha i \nu \omega \nu^{10}$ \\
\hline & $" / 30$ & $\mathrm{EY} \Delta \mathrm{OKH} \Sigma \mathrm{A}$ & $\eta \cup \delta о к \eta \sigma \alpha$ & $\mathrm{D}^{\mathrm{C}}$ & B $v l$ n.e. & $\epsilon \cup ̉ \delta o ́ \kappa \eta \sigma \alpha^{11}$ \\
\hline 1,15 & $286 \mathrm{~b} / 6$ & BABALI $\Lambda$ EIA & $\beta \alpha \sigma \iota \lambda \in \iota \alpha^{12}$ & $D^{\text {s.m. }}$ & & $\beta \alpha \sigma \iota \lambda \in i \alpha$ \\
\hline 1,17 & $" / 13$ & $\mathrm{AN} \Theta \mathrm{P} \Omega \Pi \Omega$ & & corr. & & $\dot{\alpha} \nu \theta \rho \omega ́ \pi \omega \nu^{13}$ \\
\hline 1,19 & $" / 15$ & ПРО & $\pi \rho \circ \beta \alpha \sigma$ & $\mathrm{D}^{\mathrm{s.m} .}$ & B $v l$ n.e. & $\pi \rho о \sigma \beta \alpha^{\prime} \varsigma^{14}$ \\
\hline 1,22 & $" / 26$ & $\begin{array}{l}\mathrm{EX} \Omega \mathrm{N} \text { OYX } \Omega \Sigma \text { OI } \\
\text { ГPAMMA } \cdot \mathrm{EI} \Sigma\end{array}$ & 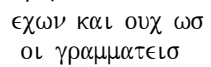 & $\begin{array}{l}\mathrm{D}^{\mathrm{B}} \\
\text { Scr. }^{15}\end{array}$ & B $v l$ n.e. & 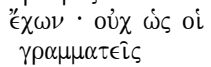 \\
\hline 1,24 & $" / 30$ & АПО $\Lambda \mathrm{E} \Sigma \mathrm{A} O \Lambda \mathrm{E} \Sigma \mathrm{AI}$ & & corr. & & $\dot{\alpha} \pi 0 \lambda \epsilon^{\prime} \sigma \alpha \iota^{16}$ \\
\hline 1,27 & $287 \mathrm{~b} / 6$ & 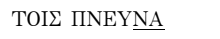 & $\tau O\left\llcorner\sigma \pi \nu \in U \nu^{17}\right.$ & $\mathrm{D}^{\mathrm{s} . \mathrm{m} .}$ & & 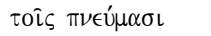 \\
\hline 1,34 & $" / 25$ & $\mathrm{HI} \Delta \mathrm{I} \Sigma \mathrm{AN}$ & $\eta \delta\llcorner\sigma \alpha \bar{\nu}$ & $\mathrm{D}^{\mathrm{s} . \mathrm{m}}$ & & $\eta ̣$ \\
\hline 1,35 & $" / 30$ & 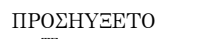 & & cj. $^{18}$ & (B $v l$ n.e.) & 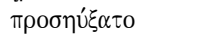 \\
\hline 1,36 & $" / 31$ & $\mathrm{TO}^{\mathrm{TE}}$ & & $\mathrm{D}^{\mathrm{p} . \mathrm{m} .}$ & & $\tau o ́ \tau \epsilon^{19}$ \\
\hline 1,40 & $288 \mathrm{~b} / 6$ & $\begin{array}{l}\text { EAN } \Theta E \Lambda E I \Sigma \\
\triangle \text { YNA } \Sigma A I\end{array}$ & & corr. & B $v l$ n.e. & 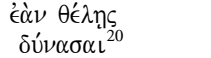 \\
\hline 1,43 & $" / 10$ & ENEBPILAMENO $\Sigma$ & & corr. & & $\epsilon \mu \beta \rho\left\llcorner\sigma \alpha^{\prime} \mu \epsilon \nu o \varsigma^{21}\right.$ \\
\hline 2,2 & $" / 22$ & $\Sigma \mathrm{YNHK} \Theta \mathrm{H} \Sigma \mathrm{AN}$ & $\sigma u \nu \eta \chi \theta \eta \sigma \alpha \nu$ & $\mathrm{D}^{\mathrm{A}}$ & & $\sigma u \nu \eta \dot{\chi} \chi \theta \eta \sigma \alpha \nu^{22}$ \\
\hline 2,7 & $289 \mathrm{~b} / 5$ & 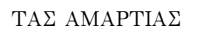 & $\alpha \mu \alpha \rho \tau \iota \alpha \sigma$ & $\mathrm{D}^{\mathrm{s.m} .}$ & B $v l$ n.e. & $\tau \grave{\alpha} \varsigma \dot{\alpha} \mu \alpha \rho \tau \iota^{\prime} \alpha \varsigma$ \\
\hline 2,9 & $" / 9$ & ПАРА $\Lambda$ YТ $\Omega$ & & b.1. ? $?^{23}$ & B $v l$ n.e. & $\pi \alpha \rho \alpha \lambda u^{\prime} \tau \omega$ \\
\hline 2,13 & $" / 22$ & ПАЕ OХ $\Lambda O \Sigma$ & 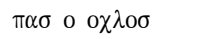 & $\mathrm{D}^{\mathrm{A}}$ & B $v l$ n.e. & $\pi \hat{\alpha} \varsigma$ oै $\chi \lambda \sigma^{24}$ \\
\hline
\end{tabular}

9 Confusion du copiste latin dûe à la forme féminine du nominatif et de l'accusatif, 'Iopó́́ $\nu \eta \varsigma$, ou : voir « Les variants » $\mathrm{I}, \mathrm{n}^{\mathrm{o}} 24$.

10 Malgré la forme neutre du tò $\pi \nu \in \hat{u} \mu \alpha$, D05 le fait concerter toujours avec le pronom masculin pour indiquer son caractère personnel : « Les variants » $\mathrm{I}, \mathrm{n}^{\circ} 27$.

11 « $\epsilon \mathrm{U}-$ wird im Att. mit $\eta \cup-$ augmentiert ..., später lieber mit $\epsilon \mathrm{U}-$, das auch im NT überwiegt » (B-D-R, 67, 1c et n. 2)

12 Erreur par dittographie.

13 Omission d'un tilde sur l' $\Omega$ à la fin de la ligne.

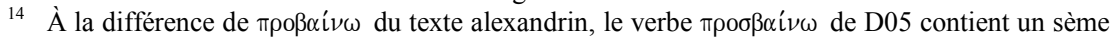
de rapprochement : voir « Les variants » II, $\mathrm{n}^{\circ} 46$

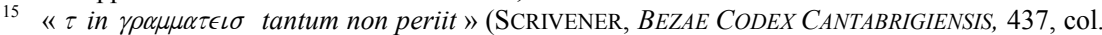
$3)$.

16 Erreur par dittographie.

17 « $\pi \in \underline{v \nu \alpha}$ has been altered by a later hand to $\underline{\pi \nu \in U \nu} »$ (PARKER, Codex Bezae, 296).

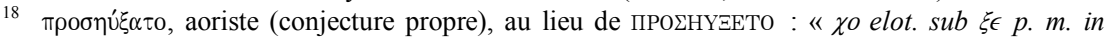

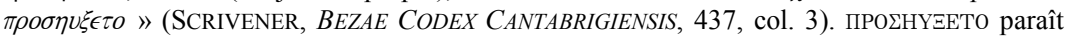

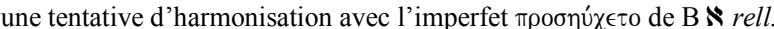

19 Érreur corrigée par la p.m.

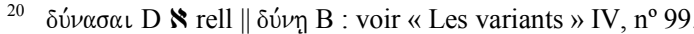

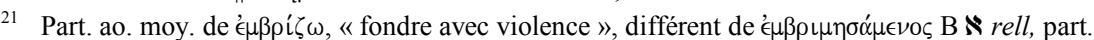

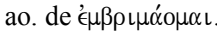

22 Par dissimilation.

23 La $v l \pi \alpha \rho \alpha \lambda \cup ́ \tau \omega$, dans la bouche de Jésus, pourrait être correcte (par substantivation de $\pi \alpha-$ $\rho \alpha ́ \lambda \cup \tau 0 \varsigma$, o, ov, «paralysé »). Dans les autres allusions au paralytique le narrateur utilise l'ad-

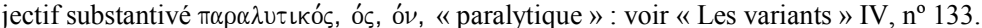

24 Le manque d'article dans D05 indique que cette « foule» n'a rien à voir avec « la foule » mentionnée auparavant en 2,4: voir « Les variants » IV, $\mathrm{n}^{\circ} 141$. 
Le Codex de Bèze : base indispensable pour une édition de Marc

\begin{tabular}{|c|c|c|}
\hline 2,21 & $290 \mathrm{~b} / 20$ & PAKKOY \\
\hline 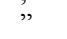 & $" / 24$ & XEIP $\Omega N \quad \Sigma X I \Sigma M A$ \\
\hline 3,2 & $291 b / 15$ & AYTON \\
\hline 3,3 & $" / 17$ & EEHPAMENHN \\
\hline 3,14 & $292 b / 21$ & 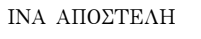 \\
\hline 3,17 & $" / 28$ & BOANEPГНऽ \\
\hline 3,18 & $" / 32$ & TO KANANAION \\
\hline$"$ & $" / 33$ & IOY $\triangle \mathrm{A} \Sigma \Sigma \mathrm{KAPI} \Omega \Theta$ \\
\hline 3,21 & $293 \mathrm{~b} / 5$ & ГРАMMATEIN \\
\hline 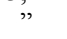 & $" / 6$ & 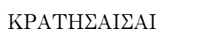 \\
\hline$"$ & $" / 7$ & 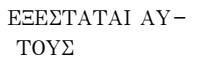 \\
\hline 3,26 & $" / 19$ & 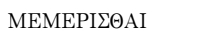 \\
\hline 3,28 & $" / 27$ & 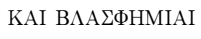 \\
\hline 4,1 & $294 b / 15$ & 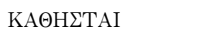 \\
\hline 4,5 & $" 25$ & EEANE $\Sigma T E I \Lambda E N$ \\
\hline 4,12 & $295 b / 13$ & $\Sigma \mathrm{YN} \Omega \Sigma \mathrm{IN}$ \\
\hline$"$ & $" / 14$ & АФЕ $\Theta Н \Sigma O M A I$ \\
\hline 4,16 & $" / 25$ & $\begin{array}{l}\text { TA ПЕTP } \Omega \Delta \mathrm{H} \Sigma \\
\Sigma \Pi \text { IIPOMENOI }\end{array}$ \\
\hline
\end{tabular}

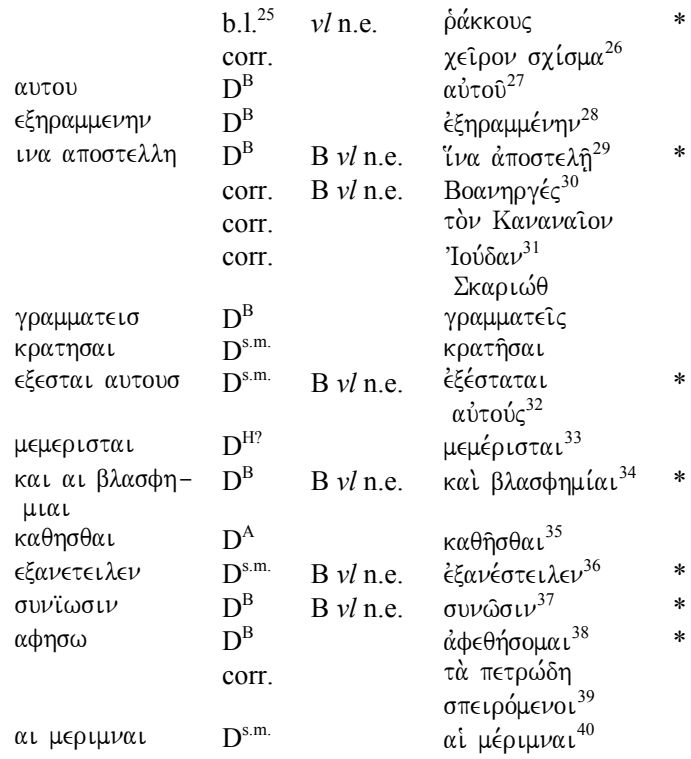

25 La même réduplication figure dans Mt 9,16 D. Sans réduplication $\mathrm{B} \aleph a l$.

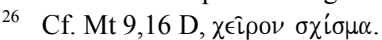

27 L'accusatif AYTON est dû probablement à l'influence du latin accusarent eum, le scribe étant un latin.

28 Cf. Mc 11,20 D. Voir « Les variants » V, nº 188.

29 «the future (indicative) usually denotes a lasting state » (WINER, 361).

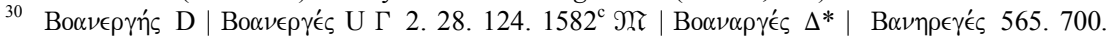

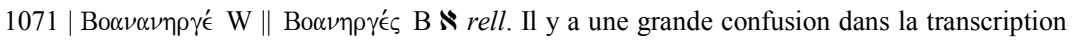
de l'hébreu.

31 La même erreur se présente dans la page latine, Simonem Cananeum et Iudas Scarioth.

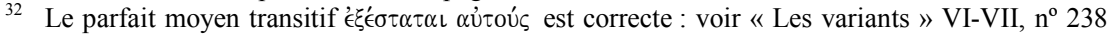
(pour la n. 25, lire « Mayser I/II », au lieu de « Mayser II/I »). Dans le texte Césaréen $\left(\Theta f^{13}\right.$

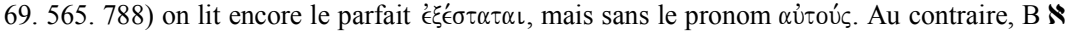

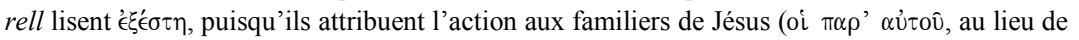

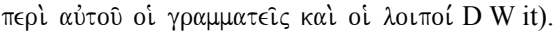

33 Par dissimilation.

34 L'omission de l'article pourait être intentionnée : voir « Les variants » VI-VII, n 251

35 Par dissimilation progressive.

36 L'aoriste 2 de ${ }^{\prime} \xi \xi \nu \alpha \sigma \tau^{\prime} \hat{\epsilon} \lambda \lambda \omega$, au sens transitif, est correcte : voir « Les variants » VI-VII, $\mathrm{n}^{\circ} 288$.

$37 \sigma \cup \nu \hat{\omega} \sigma \iota \nu$ (subjonctif aoriste) D L W 1. 565. 1582*. 1071. $1424 \| \sigma \cup \nu \iota \hat{\omega} \sigma \iota \nu$ (subjonctif présent) B $\aleph$ rell : cf. URBAN, «Intercambios vocálicos», 266.

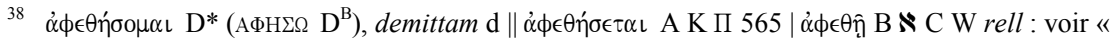
Les variants » VI-VII, n 305.

39 Erreur par dittographie.

40 Sous l'influence du latin errores? 


\begin{tabular}{|c|c|c|c|c|c|c|}
\hline 4,24 & $" / 18$ & TA AKOYETE & & b..$^{41}$ & B $v l$ n.e. & $\tau \grave{\alpha} \dot{\alpha}$ кои́є $\tau \epsilon$ \\
\hline 4,25 & $" / 20$ & AN EXEI & $\alpha \nu \in \chi \eta$ & corr. & B $v l$ n.e. & $\grave{\alpha}^{\nu}{ }^{\prime} \chi \chi \eta^{42}$ \\
\hline 4,31 & $297 b / 3$ & O OTIAN & $0 \sigma 0 \tau \alpha \nu$ & $\mathrm{D}^{\mathrm{H}}$ & B $v l$ n.e. & ö ǒ $\tau \alpha \nu^{43}$ \\
\hline$"$ & $" \quad$, & $\begin{array}{l}\text { O...MEIKPOTEPON } \\
\text { ELTIN }\end{array}$ & $\begin{array}{l}\operatorname{o\sigma }_{\mu \in \nu} \ldots \mu \epsilon \iota \kappa \rho о \tau \epsilon \rho о \sigma \\
\epsilon \sigma \tau \iota \nu\end{array}$ & $\mathrm{D}^{\mathrm{C} ?}$ & & 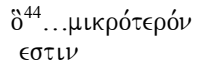 \\
\hline 4,32 & $" / 5$ & MEIZ $\Omega N$ & & b. $1 . .^{45}$ & & $\mu \in i \zeta \omega \nu$ \\
\hline 4,34 & $" / 12$ & $\mathrm{KA \Theta} \mathrm{I} \triangle \mathrm{IAN}$ & & corr. & & $\kappa \alpha \tau^{\prime} i \delta i \alpha \nu^{46}$ \\
\hline$"$ & ", & EПЕ $\Lambda \mathrm{YE}$ & $\epsilon \pi \in \lambda \cup \in \mathcal{V}$ & $\mathrm{D}^{\mathrm{B}}$ & & $\mathfrak{\epsilon}^{\prime} \pi^{\prime} \in \lambda \cup \in \nu$ \\
\hline 4,35 & $" / 14$ & $\triangle \mathrm{IE} \Lambda \Theta \Omega N M E N$ & & corr. & & $\delta \iota^{\prime} \lambda \theta \omega \mu \epsilon \nu$ \\
\hline 4,36 & $" / 16-17$ & $\begin{array}{l}\mathrm{A} \Lambda \Lambda \mathrm{AI} \Delta \mathrm{E} \text { П } \Lambda \mathrm{OIAI} \\
\Pi \mathrm{O} \Lambda \Lambda \mathrm{AI}\end{array}$ & $\begin{array}{l}\alpha \lambda \lambda \alpha \delta \epsilon \pi \lambda \circ\llcorner\alpha \\
\pi 0 \lambda \lambda \alpha\end{array}$ & $\mathrm{D}^{\text {s.m. }}$ & B $v l$ n.e. & $\begin{array}{l}\alpha^{\prime} \lambda \lambda \alpha \iota \delta^{\prime} \epsilon \pi \lambda \circ \hat{\epsilon} \alpha \iota \\
\pi 0 \lambda \lambda \alpha \alpha^{\prime 7}\end{array}$ \\
\hline 5,4 & $298 b / 12$ & ILXYN & I $\sigma \chi \cup \iota \nu$ & $\mathrm{D}^{\text {s.m. }}$ & & 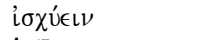 \\
\hline 5,8 & $" / 21$ & $\mathrm{O} \underline{\mathrm{IHY}}$ & 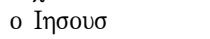 & $\mathrm{D}^{\mathrm{A}}$ & & 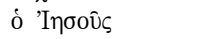 \\
\hline 5,14 & $299 b / 4$ & AYTOE & $\alpha \cup \tau O u \sigma$ & $\mathrm{D}^{\mathrm{B}}$ & & 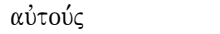 \\
\hline 5,19 & $" / 18$ & $\underline{\mathrm{IIH} \Sigma}$ & Inбous & $\mathrm{D}^{\mathrm{s.m} .}$ & & 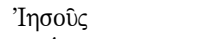 \\
\hline 5,27 & $300 \mathrm{~b} / 6$ & $\overline{\Pi \mathrm{E}}$ & $\pi \epsilon \rho \iota$ & $\mathrm{D}^{\mathrm{A}}$ & & $\pi \epsilon \rho i ́$ \\
\hline 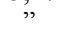 & $" / 7$ & 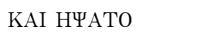 & $\eta \psi \alpha \tau 0$ & $\mathrm{D}^{\mathrm{s} . \mathrm{m} .}$ & B $v l$ n.e. & $\kappa \alpha i ~ \dddot{\psi} \psi \alpha \tau 0^{48}$ \\
\hline 5,30 & $" / 15$ & $\begin{array}{l}\text { THN } \triangle \text { YNAMIN } \\
\text { EEE } \Lambda \Theta O Y \Sigma A N\end{array}$ & 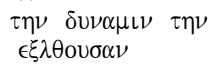 & $\mathrm{D}^{\mathrm{E}}$ & $v l$ n.e. & 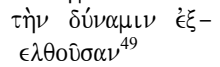 \\
\hline 5,41 & $301 b / 17$ & $\begin{array}{l}\text { PABBI @ABITA } \\
\text { KOYMI }\end{array}$ & 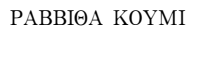 & $\begin{array}{l}\text { cj. de } \\
\text { Wel }^{50}\end{array}$ & & 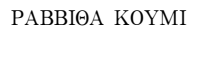 \\
\hline 6,1 & $" / 25$ & KАПЕ $\Lambda \Theta E N$ & & corr. & & $\kappa \alpha \grave{\iota} \dot{\alpha} \pi \hat{\eta} \lambda \theta \epsilon \nu^{51}$ \\
\hline 6,3 & $302 b / 1$ & OYK OYT $\Omega \Sigma$ & 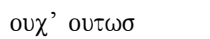 & $\mathrm{D}^{\mathrm{A}}$ & & oủ $\chi$ oü $\tau \omega \varsigma^{52}$ \\
\hline 6,4 & $" / 8$ & $\begin{array}{l}\text { EN TYI } \Sigma^{53} \Sigma Y N- \\
\text { ГENELI }\end{array}$ & $\begin{array}{l}\epsilon \nu \quad \tau 0\llcorner\sigma \quad \sigma \nu \nu \gamma \epsilon- \\
\nu \in \cup \sigma \overline{\mathrm{i}} / \sigma \cup \gamma \gamma \epsilon \nu \in \sigma \iota\end{array}$ & $\begin{array}{l}\mathrm{D}^{\mathrm{A}} \\
\text { corr. }\end{array}$ & B $v l$ n.e. & 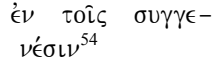 \\
\hline
\end{tabular}

41 L'interrogation indirecte $\tau \alpha \dot{\alpha}$ au lieu du pronom $\ddot{\alpha}$ est correcte : voir « Les variants » VI-VII, $\mathrm{n}^{\mathrm{o}}$ 329.

42 D05, à la différence du texte alexandrin, parle d'une éventualité : voir « Les variants » VI-VII, $\mathrm{n}^{\mathrm{o}} 331$.

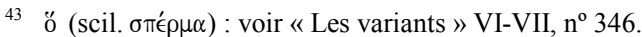

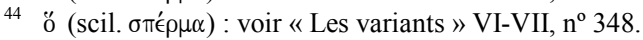

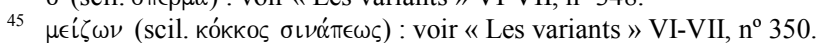

46 Par aspiration devant une voyelle.

47 À nouveau le Codex de Bèze change le genre neutre de $\pi \lambda \circ \hat{\imath} \alpha$ par le féminin pluriel $\pi \lambda$ oî $\alpha \iota$ en indiquant qu'ici il ne s'agit pas simplement de «barques » mais de « communautés rassemblées dans les barques » : voir « Les variants » VIII, $n^{\circ} 357$.

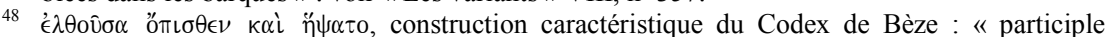

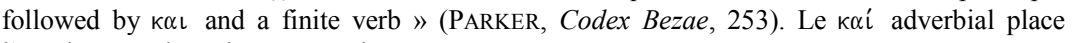
l'emphase sur le verbe personnel.

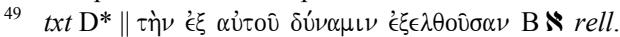

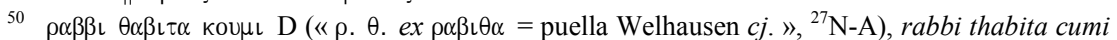

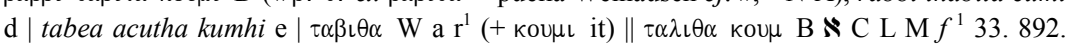
1071. 1241. 1424. 2427 al co $\mid \tau \alpha \lambda \iota \theta \alpha$ коинь A K N U $\Delta \Theta \Pi 0126 f^{13} 565.579 .700$ M q vg sy ${ }^{\text {h }}$.

51 Par crase.

52 Par dissimilation.

53 «I think that p.m. wrote $\tau \cup \iota \sigma$, not $\tau \alpha \iota \sigma »$ (PARKER, Codex Bezae, 296). 
Le Codex de Bèze : base indispensable pour une édition de Marc

\begin{tabular}{|c|c|c|c|c|c|c|}
\hline 6,13 & 303b/1 & $\mathrm{AP} \Omega \Sigma T O Y \Sigma$ & & corr. & & $\alpha \rho \rho \omega ́ \sigma \tau o u \varsigma^{55}$ \\
\hline 6,18 & $" / 18$ & $\begin{array}{l}\text { ¿E EXEIN AY | } \\
\text { THN TYNAIKA }\end{array}$ & 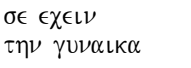 & $\mathrm{D}^{\mathrm{s} . \mathrm{m}} \cdot ?^{56}$ & B $v l$ n.e. & 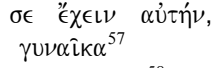 \\
\hline 6,19 & $" / 22$ & OYX HAYNATO & 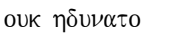 & $\mathrm{D}^{\mathrm{A}}$ & & 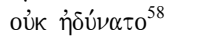 \\
\hline 6,21 & $" / 28$ & $\mathrm{KAI} \ldots \Delta \mathrm{E}$ & $\kappa \alpha \iota$ & $\mathrm{D}^{\text {s.m. }}$ & B $v l$ n.e. & $\kappa \alpha i \ldots \delta \epsilon^{59}$ \\
\hline$"$ & $" / 29$ & 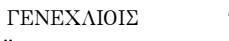 & $\gamma \in \nu \in \theta \lambda \iota\llcorner$ เ $\sigma$ & $\mathrm{D}^{\mathrm{A}}$ & & $\gamma \in \nu \in \theta \lambda i ́ o \iota \varsigma$ \\
\hline 6,25 & $304 b / 12$ & † $\Omega$ ANOY & & corr. & B $v l$ n.e. & 'I $\omega \alpha \dot{\alpha} \nu \nu \mathrm{ou}^{60}$ \\
\hline 6,27 & $" / 17$ & ¿ПЕКОААТОРАN & $\sigma \pi \epsilon \kappa о \lambda \alpha \tau о \rho \alpha$ & $\mathrm{D}^{\mathrm{s.m} .}$ & & $\sigma \pi \epsilon \kappa o \cup \lambda \alpha^{\prime} \tau o \rho \alpha^{61}$ \\
\hline 6,31 & $" / 33$ & EYKAIPOE EIXON & $\epsilon \cup \kappa \alpha\llcorner\rho \omega \sigma \epsilon\llcorner\chi O \nu$ & $\mathrm{D}^{\mathrm{B}}$ & & 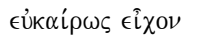 \\
\hline 6,33 & $305 \mathrm{~b} / 5$ & ПANT $\Omega N$ ПO $\Lambda \mathrm{E} \Omega N$ & & $\mathrm{cj}^{62}$ & & $\begin{array}{c}\pi \alpha<\sigma \hat{\omega}>\nu \\
\pi \hat{\lambda} \in \omega \nu\end{array}$ \\
\hline 6,35 & $" / 13-14$ & $\begin{array}{l}\text { TOПО } \mid \\
\mathrm{H} \Delta \mathrm{H} \Omega \mathrm{PA} \Pi \mathrm{\Pi O} \Lambda \mathrm{H}\end{array}$ & 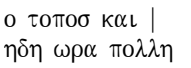 & $\mathrm{D}^{\mathrm{B}}$ & B $v l$ n.e. & 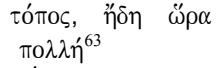 \\
\hline 6,37 & & $\Delta \Omega \Sigma \Omega \mathrm{MEN}$ & & b.1. ${ }^{64}$ & & $\delta \omega ́ \sigma \omega \mu \epsilon \nu$ \\
\hline 6,38 & $" / 22$ & EXETE $\Sigma$ & & corr. $^{65}$ & & $" \epsilon \chi \in \tau \epsilon$ \\
\hline$"$ & $" / 24$ & $\Delta Y \Omega^{66}$ & бvo & $\mathrm{D}^{\mathrm{D}}$ & B $v l$ n.e. & $\delta u^{\prime} \omega^{67}$ \\
\hline 6,45 & $306 \mathrm{~b} / 7$ & ПРОГАГЕIN AYTON & $\pi \rho \circ \alpha \gamma \in L \nu \alpha v \tau o \nu$ & $\mathrm{D}^{\mathrm{s.m} .}$ & B $v l$ n.e. & 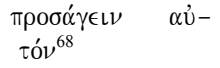 \\
\hline$"$ & $" / 8$ & $\mathrm{BH} \Sigma \Sigma \mathrm{A} I \Delta \mathrm{AN}$ & & corr. & & $\mathrm{B \eta} \theta \sigma \alpha \ddot{\mathrm{I}} \delta \propto \alpha \nu^{69}$ \\
\hline 5,51 & $" / 27$ & E $\Xi E \Sigma T A N T O$ & $\epsilon \xi \in\llcorner\sigma \tau \alpha \nu \tau 0$ & $\mathrm{D}^{\mathrm{A}}$ & & ${ }^{\prime} \epsilon \xi \dot{\prime} \sigma \tau \alpha \nu \tau o$ \\
\hline
\end{tabular}

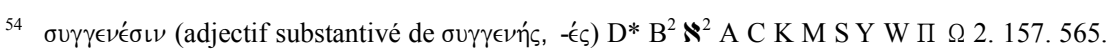

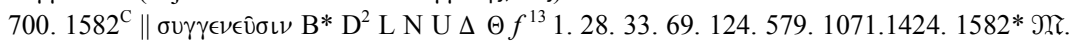

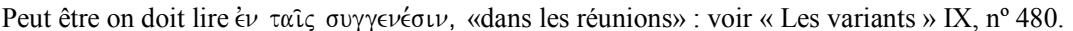

55 Les deux formes sont possibles, mais voir 6,5.

56 « $\alpha \nu$ ( $\alpha \nu$ Mill. errore) elot. ad finem » (SCRIVENER, BEZAE CODEX CANTABRIGIENSIS, 438, col. 2) : «Il ne t'est pas permis d'avoir la femme de ton frère. »

57 « Il n'est pas permis que tu aies celle-ci, étant femme de ton frère. » Cf. URBAN, « Intercambios vocálicos », 266.

58 Par aspiration devant une voyelle.

59 Voir « Les variants» X, n 514 (mais au lieu de « única », lire « raríssima » : cf. Mc 4,36).

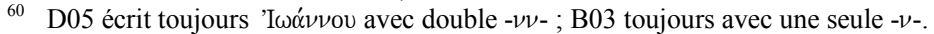

$61 \mathrm{Du}$ « lat. speculator, soldat chargé de la garde des prisionners » (Bailly, s.v.). L'accusatif $\Sigma \Pi$ IIO $\triangle \mathrm{ATOPAN}$ de $\mathrm{D}^{*}$ est dû probablement à l'influence du latin, si bien le traducteur a traduït

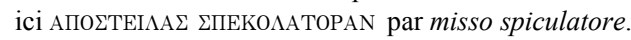

62 Erreur par haplographie (conjecture propre).

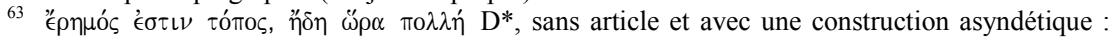
voir « Les variants » XI, $\mathrm{n}^{\text {os }} 557-558$

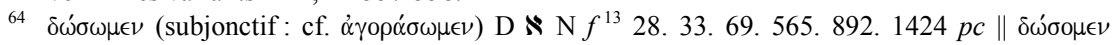
(futur) B $\mathfrak{P}^{45} \mathrm{~A} \mathrm{~L} \Delta 2427.2542 p c \mid \delta \omega \mu \epsilon \nu$ (aoriste) K M U W $\Gamma \Theta \Pi f^{1} 2.157 .579 .700 .1071$ Mr.

65 La $\Sigma$ de EXETE $\Sigma$ pourrait être dûe à l'influence du latin habetis.

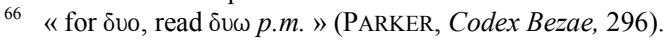

67 «les deux finales -o et - $\omega$ sont également anciennes » (BAILLY, s.v.).

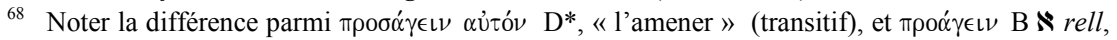

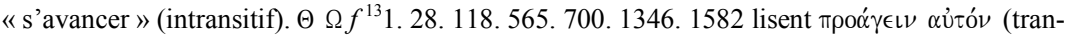
sitif : une conflation).

69 Par assimilation. 


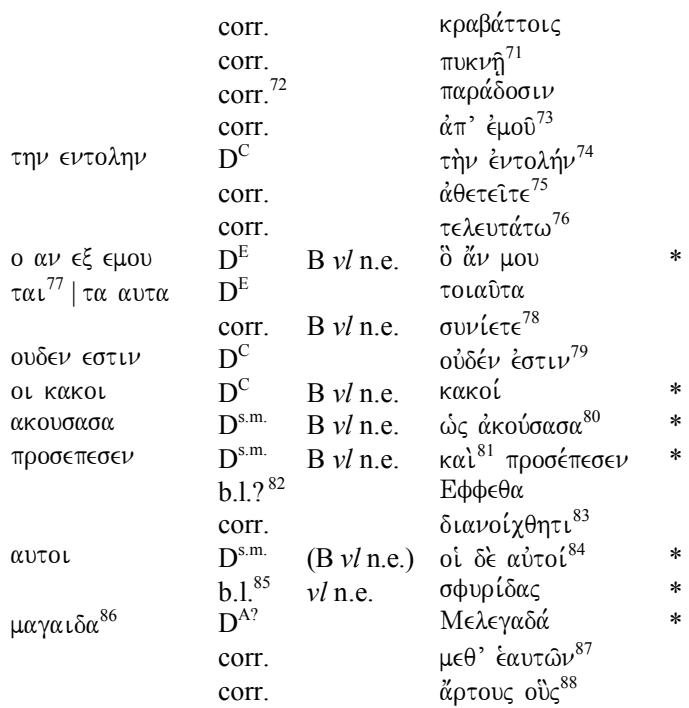

70 Par influence du latin grabattis : cf. 2,4.9.11.12.

71 " $\gamma$ elot. sub $\kappa$ in $\pi U \kappa \mu \eta$ p. m. " (SCRIVENER, BEZAE CODEX CANTABRIGIENSIS, 438, col. 2).

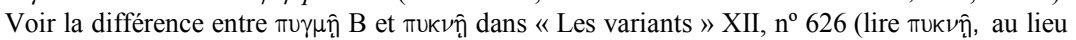

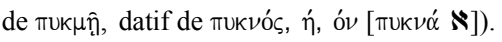

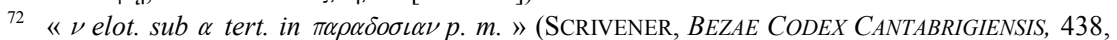
col. 2). Le copiste ( $p . m$.) avait écrit premièrement ПAPA $\triangle O \Sigma I N N$ et avait corrigé par erreur en ПAPA OOIIAN. Voir vV. 5, 8, 9.

73 Par aspiration devant une voyelle.

74 Par haplographie.

75 Par métathèse.

76 « La lectura es exclusiva de D » (URBÁN, « Intercambios vocálicos », 267).

77 «1. 24 ad finem addit $\tau \alpha \iota \mathrm{E} »$ (SCRIVENER, BEZAE CODEX CANTABRIGIENSIS, 438, col. 3).

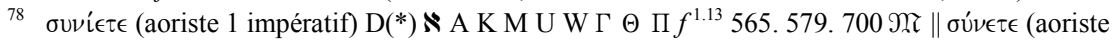
2 impératif) B H L $\Delta 1424$.

79 Par haplographie.

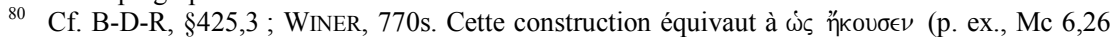
D).

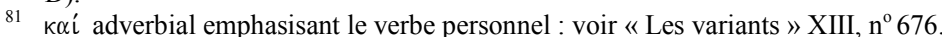

$82 \mathrm{E} \phi \phi \epsilon \theta \alpha \mathrm{D} \aleph^{2} \mathrm{~W} \| \mathrm{E} \phi \phi \alpha \theta \alpha \mathrm{B} \aleph *$ rell.

83 Par dissimilation et échange de -Y- au lieu de -OL-.

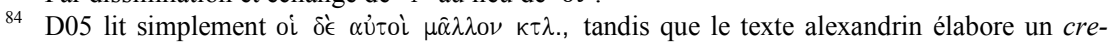

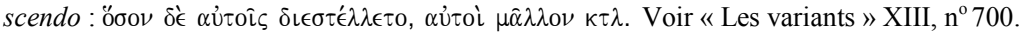

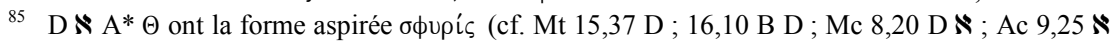
[D lac.]), tandis que le texte alexandrin lit $\sigma$ mupíc.

86 Ex lat. magidan d5?

87 Par dissimilation. 
Le Codex de Bèze : base indispensable pour une édition de Marc

\begin{tabular}{|c|c|c|c|c|c|c|c|}
\hline 8,20 & $" / 18$ & $\Sigma \Phi \mathrm{YPI} \Delta \mathrm{A} \Sigma$ & & b.1. ${ }^{89}$ & $v l$ n.e. & $\sigma \phi \cup \rho i ́ \delta \alpha \varsigma$ & $*$ \\
\hline 8,21 & $" / 20$ & ¿YNNOEITE & $\nu 0 \epsilon L \tau \epsilon$ & $\mathrm{D}^{\mathrm{s.m} .}$ & B $v l$ n.e. & $\sigma U \nu \nu 0 \epsilon \hat{\imath} \tau \epsilon^{90}$ & $*$ \\
\hline 8,35 & $314 b / 1$ & 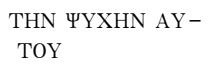 & $\begin{array}{l}\tau \eta \nu \\
\tau 0 u\end{array}$ & $\mathrm{D}^{\mathrm{A}}$ & $\mathrm{B}^{91}$ & 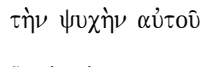 & $*$ \\
\hline 8,37 & $" / 6$ & Н TI ГАР & $\eta \tau \iota$ & $\mathrm{D}^{\mathrm{s} . \mathrm{m} .}$ & $\begin{array}{l}\text { A C } \Theta \\
v l \text { n.e. }\end{array}$ & $\grave{\eta} \tau \hat{\imath} \gamma \alpha \dot{\alpha} \rho$ & $*$ \\
\hline 9,1 & $" / 14$ & TINE $\Omega \Delta \mathrm{E}$ T $\Omega \mathrm{N}$ & $\begin{array}{l}\tau \iota \nu \in \sigma \tau \omega \nu \\
\tau \iota \nu \in \sigma \tau \omega \nu \omega \delta \epsilon\end{array}$ & $\begin{array}{l}\mathrm{D}^{\mathrm{s} . \mathrm{m} \cdot \mathrm{H}} \\
\mathrm{D}^{\mathrm{B} 93}\end{array}$ & & $\tau \iota \nu \grave{\epsilon} \varsigma \hat{\omega} \delta \epsilon \tau \hat{\omega} \nu^{94}$ & \\
\hline 9,2 & $" / 21$ & 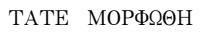 & & corr. & & $\mu \in \tau \epsilon \mu о \rho \phi \omega^{\prime} \theta \eta$ & \\
\hline 9,3 & $" / 23$ & EГENENONTO & & corr. & & $\epsilon^{\prime} \gamma^{\prime} \in{ }^{\prime} \nu \nu \tau 0^{95}$ & \\
\hline$"$ & $" / 24$ & $\Lambda \mathrm{IA}$ & $\lambda\llcorner\alpha \nu$ & $\mathrm{D}^{\mathrm{B}}$ & & $\lambda i \alpha \nu$ & \\
\hline 9,7 & $315 b / 3$ & AYTOY | AYTOY & $\rho^{96} \alpha \cup \tau o u$ & $\mathrm{D}^{\mathrm{sm} ?}$ & B $v l$ n.e. & 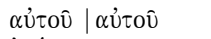 & $*$ \\
\hline 9,13 & $" / 21$ & $\mathrm{E} \Lambda \mathrm{H} \Lambda \mathrm{Y} \Theta \mathrm{E}$ & $\epsilon \lambda \eta \lambda \cup \theta \epsilon \nu$ & $\mathrm{D}^{\mathrm{D}}$ & & $\epsilon^{\prime} \lambda \eta^{\prime} \lambda \cup \theta \epsilon \nu$ & \\
\hline 9,18 & $316 \mathrm{~b} / 2$ & PA $\Sigma \Sigma E I$ & & & $v l$ n.e. & 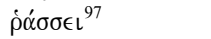 & $*$ \\
\hline 9,25 & $" / 25$ & $\mathrm{E} \Xi \mathrm{E} \Lambda \Theta \mathrm{E} \Lambda \Theta \mathrm{E}$ & $\epsilon \xi \in \lambda \theta \epsilon$ & $D^{\text {s.m. }}$ & & ${ }^{\prime} \xi \xi \in \lambda \theta \epsilon^{98}$ & \\
\hline 9,28 & $" / 33$ & KATIAN & & corr. & & $\kappa \alpha \tau^{\prime} i \delta i \alpha \nu^{99}$ & \\
\hline 9,29 & $317 \mathrm{~b} / 3$ & EN OY $\triangle \mathrm{EN}$ & & corr. & & 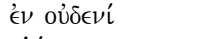 & \\
\hline 9,31 & $" / 8$ & 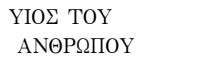 & 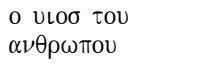 & $\mathrm{D}^{\mathrm{A}}$ & B $v l$ n.e. & 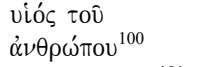 & $*$ \\
\hline 9,36 & $" / 21$ & ANAK $\Lambda \mathrm{I} \Sigma \mathrm{AMENO} \Sigma$ & $\epsilon \nu \alpha \nu \kappa \alpha \lambda \iota \sigma \alpha \mu \epsilon \nu \circ \sigma$ & $\mathrm{D}^{\mathrm{C}}$ & B $v l$ n.e. & ${ }^{\alpha} \nu \alpha \kappa \lambda \iota \sigma \alpha^{\prime} \mu \epsilon \nu \circ \varsigma^{101}$ & $*$ \\
\hline 9,41 & $318 \mathrm{~b} / 5$ & АПО $\Lambda \mathrm{E} \Sigma \mathrm{EI}$ & & b.1. ${ }^{102}$ & $v l$ n.e. & $\alpha \dot{\alpha} \pi 0 \lambda \epsilon ́ \sigma \epsilon l$ & $*$ \\
\hline
\end{tabular}

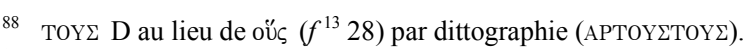

89 Voir supra, 8,8 .

$90 \nu$

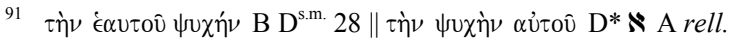

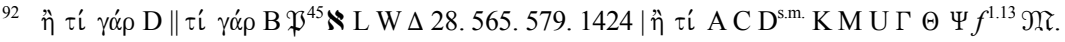

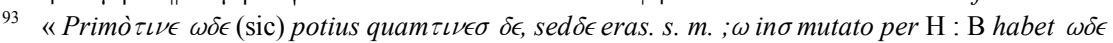
supra lineam ante $\epsilon \sigma \tau \eta \kappa o \tau \omega \nu »$ (SCRIVENER, BEZAE CODEX CANTABRIGIENSIS, 438, col. 3 [d'après PARKER, Codex Bezae, 296, « the whole correction is by $\mathrm{B} »]$ ).

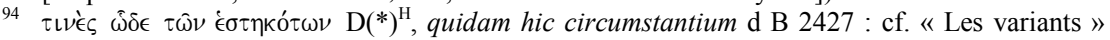
XIV, n ${ }^{\circ} 799$; PARKER, Codex Bezae, 145: «For the question of the attribution of this correction, see I. A. MoIR, ' The Reading of Codex Bezae (D-05) at Mark ix. 1 ', NTS 20 (1974), 105 ; H. GreEven, ' Nochmals Mk ix. 1 in Codex Bezae (D, 05) ', NTS 23 (1977), 305-8. »

95 Par dittographie.

96 « avtov elot. ad finem lineae » (SCRIVENER, BEZAE CODEX CANTABRIGIENSIS, 438, col. 3). La répétition du pronom souligne que c'est seulement à Jésus à qui ils doivent écouter : voir « Les variants » $\mathrm{XV}, \mathrm{n}^{\circ} 813$.

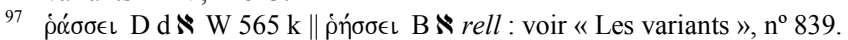

98 Erreur par dittographie.

99 Erreur par haplographie.

100 Outre l'explication donnée dans « Les variants » XVI, $\mathrm{n}^{\circ} 870$, on pourrait suggérer aussi que l'absence de l'article soit dûe à la volonté de souligner le sort du Fils de l'homme parmi les

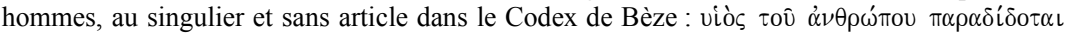
$\epsilon i \varsigma$ $\chi \in \hat{\imath} \rho \alpha \varsigma \dot{\alpha} \nu \theta \rho \omega \dot{\pi}$ ov.

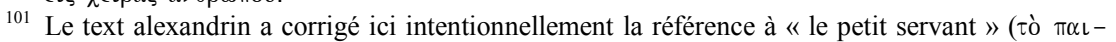

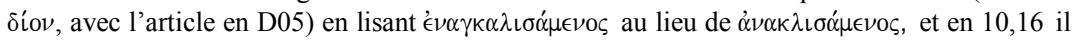

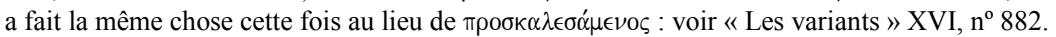




\begin{tabular}{|c|c|c|c|c|c|c|}
\hline 9,43 & $" / 13$ & EEE $\Lambda \Theta E I N$ & $\epsilon \iota \sigma \in \lambda \theta \in \iota \nu$ & $\mathrm{D}^{\mathrm{A} 103}$ & & $\epsilon \mathfrak{i} \sigma \in \lambda \theta \in \hat{\imath} \nu$ \\
\hline 9,48 & $" / 31$ & $\Sigma \mathrm{K} \Omega \Lambda \mathrm{H} \Sigma$ & $\sigma \kappa \omega \lambda \eta \xi$ & $\mathrm{D}^{\mathrm{A} ?}$ & & $\sigma \kappa \omega^{\prime} \lambda \eta \xi$ \\
\hline 10,1 & $319 b / 4$ & 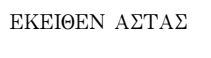 & $\epsilon \kappa \in L \theta \in \nu \quad \alpha \nu \alpha \sigma \tau \alpha \sigma$ & $\mathrm{D}^{\mathrm{C}}$ & & 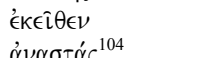 \\
\hline 10,3 & $" / 12$ & YMIN ETEI $\Lambda$ ATO & $u \mu L \nu \in \nu \in \tau \epsilon \iota \lambda \alpha \tau o$ & $\mathrm{D}^{\mathrm{C}}$ & & $\begin{array}{l}\alpha \nu \alpha \sigma \tau \alpha \zeta \\
\dot{u} \mu \hat{\imath} \nu \\
\dot{\epsilon} \nu \in \tau \epsilon \dot{i} \lambda \alpha \tau 0^{105}\end{array}$ \\
\hline 10,6 & $" / 18$ & $\Theta H \Lambda Y N$ & $\theta \eta \lambda u$ & $\mathrm{D}^{\mathrm{s.m}}$ & B $v l$ n.e. & $\theta \hat{\eta} \lambda \cup \nu^{106}$ \\
\hline 10,10 & $" / 26$ & EIEIL TIN OIKIAN & 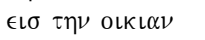 & $D^{s . m \cdot C}$ & & 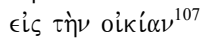 \\
\hline 10,14 & $320 \mathrm{~b} / 4$ & ПАI $\triangle \mathrm{APIA}$ & $\pi \alpha\llcorner\delta\llcorner\alpha$ & $\mathrm{D}^{\mathrm{s.m}}$ & B $v l$ n.e. & $\pi \alpha\left\llcorner\delta \alpha ́ \rho\left\llcorner\alpha^{108}\right.\right.$ \\
\hline 10,25 & $321 b / 5$ & $\begin{array}{l}\text { EILE } \Lambda \text { EY } \Sigma O N T . \\
\text { T..EION }^{109}\end{array}$ & & cj. & & $\begin{array}{l}\epsilon i \sigma \in \lambda \in \cup ́ \sigma o \nu \tau \alpha \iota \\
\tau \dot{\alpha} \chi \in\llcorner O \nu\end{array}$ \\
\hline 10,33 & $322 b / 8$ & EANATOY & $\theta \alpha \nu \alpha \tau \omega$ & $\mathrm{D}^{\mathrm{B}}$ & B $v l$ n.e. & $\theta \alpha \nu \alpha^{\prime} \tau 0 u^{110}$ \\
\hline 10,34 & $" / 11$ & ENITY $\Xi O Y \Sigma I N$ & $\epsilon \nu \pi \tau \cup \sigma o u \sigma \iota \nu$ & $\mathrm{D}^{\mathrm{B}}$ & & $\mathfrak{\epsilon}^{\prime} \mu \pi \tau \dot{\sigma} \sigma o v \sigma \iota \nu^{111}$ \\
\hline 10,39 & $" / 29$ & ВАПТІ $\Sigma \Theta H E \Sigma \Theta A I$ & & corr. & & $\beta \alpha \pi \tau \iota \sigma \theta \eta \dot{\eta} \sigma \in \sigma \theta \epsilon^{112}$ \\
\hline 10,40 & $" / 31$ & $\mathrm{~A} \Lambda \Lambda \mathrm{OI} \Sigma$ & $\alpha \lambda \lambda \circ \circ \sigma$ & $\mathrm{D}^{\mathrm{B}}$ & & $\begin{array}{l}\dot{\alpha} \lambda \lambda \lambda^{\prime} \text { oíc/ } \\
\alpha^{\prime \prime} \lambda \lambda \operatorname{olc}^{113}\end{array}$ \\
\hline 10,42 & $323 b / 3$ & $\begin{array}{l}\text { KAIKATAKYPI- } \\
\text { EY } O Y \Sigma I N\end{array}$ & к $\alpha \tau \alpha \kappa \cup \rho\llcorner\in \cup \sigma o v \sigma \iota \nu$ & $\mathrm{D}^{\mathrm{s.m}}$ & B $v l$ n.e. & 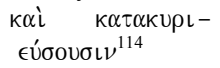 \\
\hline 10,46 & $" / 17$ & BAPITEIMIAL & & cj. & & $\mathrm{B} \alpha \rho u \tau \iota \mu i^{\prime} \alpha \varsigma^{115}$ \\
\hline 10,47 & $" / 18$ & NAZOPHNOE & $\nu \alpha \zeta \omega \rho \eta \nu о \sigma$ & $\mathrm{D}^{\mathrm{B}}$ & & 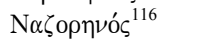 \\
\hline 11,2 & $324 b / 9$ & KAIKA@HKEN & $\kappa \alpha \iota \kappa \alpha \theta \in \iota \kappa \in \nu$ & $\mathrm{D}^{\mathrm{A}}$ & & 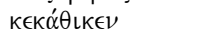 \\
\hline & & КАI АГАГЕТЕ & $\alpha \gamma \alpha \gamma \epsilon \tau \epsilon$ & $\mathrm{D}^{\mathrm{s} . \mathrm{m}}$ & B $v l$ n.e. & 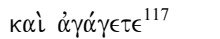 \\
\hline
\end{tabular}

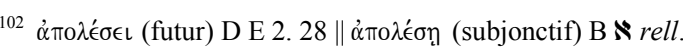

103 « the p.m. reading is $\sigma \epsilon$. Corrector A altered this to $\epsilon\llcorner\sigma »$ (PARKER, Codex Bezae, 296).

104 Par haplographie.

105 Par haplographie.

${ }^{106}$ Cf. Mt 19,4 D*. D05 se serve de la forme $\theta \hat{\eta} \lambda u \varsigma$ de l'adjectif $\theta \hat{\eta} \lambda u \varsigma, \theta \dot{\eta} \lambda \in\llcorner\alpha, \theta \hat{\eta} \lambda u$ comme féminin (cf. B-A-G, s.v. : « but also $\theta \hat{\eta} \lambda u \varsigma$ as fem. »).

107 Par dittographie et itacisme.

108 Voir « Les variants » XVI, n ${ }^{\circ} 939$.

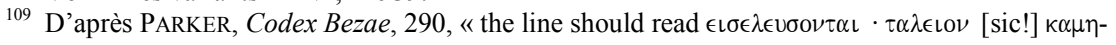
$\lambda \circ \varsigma »$.

110 Toutes les deux constructions sont valables : voir « Les variants » XVI, nº 985.

111 Par échange consonantique. D d $p c\left(\mathrm{ff}^{2}\right)(\mathrm{k})$ sont les seuls témoins qui omettent, avec raison,

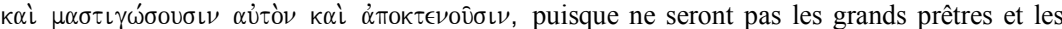
scribes qui « le flagelleront et le mettront à mort »; $\mathrm{A}^{2} 2 * .157 .1346$ ometten seulement $\kappa \alpha i$

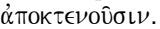

112 Par haplographie.

113 Dans l'écriture continue les deux leçons sont possibles. Le Codex de Bèze montre une préfé-

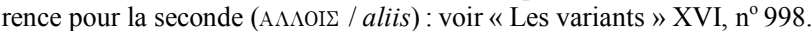

114 Voir «Les variants » XVI, $n^{\circ} 1004$. Dans cette note j'avait oublié de commenter l'omission du

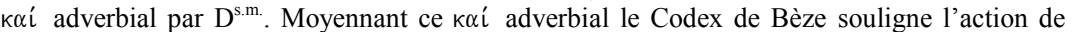
« commander en maîtres » de « ceux qu'on regarde comme les chefs des nations ».

115 Par un double échange de I pour Y et de EI pour I : voir « Les variants » XVI, n 1013.

116 Voir mon article "Nazareno" y "Nazoreo" con especial atención al Códice Bezae », en R. PIERRI (ed.), Grammatica intellectio Scripturae. Saggi filologici di greco biblico in onore di Lino Cignelli OFM, Jerusalem : Franciscan Printing Press, 2006, pp. 183-204, esp. 183-186.

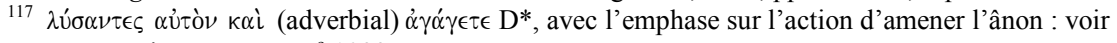
«Les variants » XVII, n ${ }^{\circ} 1033$ 


\begin{tabular}{|c|c|c|c|}
\hline 11,8 & $" / 24$ & $\Delta \mathrm{E}$ E $\Sigma T \mathrm{TIBA} \Delta \mathrm{A} \Sigma$ & $\delta \epsilon \sigma \tau \iota \beta \alpha \delta \alpha \sigma$ \\
\hline 11,10 & $" / 30$ & KAI ЕYАОГНMЕNH & $\epsilon \cup \lambda \circ \gamma \eta \mu \epsilon \nu \eta$ \\
\hline$"$ & $" / 32$ & 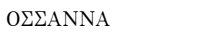 & $\omega \sigma \alpha \nu \nu \alpha$ \\
\hline 11,11 & $" / 33$ & ЕРО $О \Lambda \mathrm{YMA}$ & Iєробод $\nu \mu \alpha$ \\
\hline$"$ & $325 \mathrm{~b} / 2$ & OY $\Sigma A \Sigma \Omega$ PA $\Sigma$ & 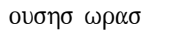 \\
\hline 11,12 & $" / 4$ & EПAYPIONON & $\epsilon \pi \alpha \cup \rho\llcorner O \nu$ \\
\hline & & & $<\alpha \cup \tau>0 \nu$ \\
\hline 11,14 & $" / 11$ & $\mathrm{E}^{\mathrm{K} \Sigma} \Xi \mathrm{OY}$ & \\
\hline 11,15 & $" / 19$ & ПЕРІ & \\
\hline 11,17 & $" / 24$ & AYTHN & $\alpha \cup \tau O \nu$ \\
\hline 11,23 & $326 \mathrm{~b} / 7$ & $\triangle \mathrm{IAKPI \Theta H \Sigma}$ & $\delta\llcorner\alpha \kappa \rho \iota \theta \eta$ \\
\hline 11,25 & $" / 13$ & EITEI & \\
\hline 11,31 & $327 b / 1$ & $\triangle \mathrm{IE} \Lambda O \Gamma I Z O N T O$ & $\delta \iota \in \lambda \mathrm{o} \iota \zeta \mathrm{o \nu}$ \\
\hline " & $" / 3$ & YMIN & $\eta \mu \iota \nu$ \\
\hline 11,32 & $" / 5$ & ФOBOYMEN & $\phi о \beta о ч \mu \epsilon \Theta \alpha$ \\
\hline 11,33 & $" / 8$ & $\mathrm{AYT} \Omega$ & \\
\hline$"$ & $" / 9$ & 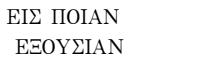 & $\epsilon \nu \pi \circ\llcorner\alpha \in \xi$ ov $\sigma\llcorner\alpha$ \\
\hline
\end{tabular}

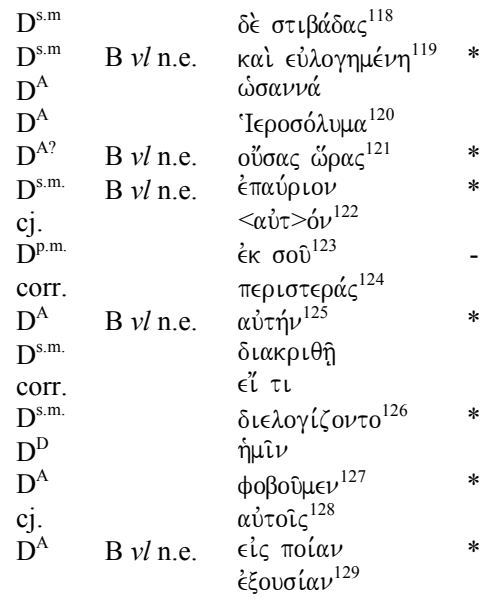

${ }_{118}$ Par dittographie.

119 D'après le Codex de Bèze, le kaí sépare clairement les cris diamétralement opposés de l'un et l'autre choeur : voir « Les variants » XVII, $n^{\circ} 1052$

${ }^{120}$ Erreur du copiste.

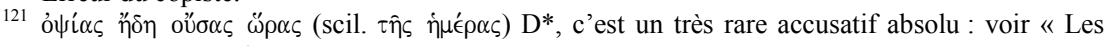
variants » XVII, $\mathrm{n}^{\circ} 1056$.

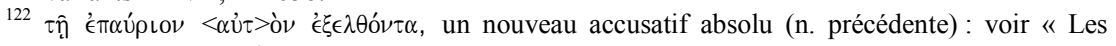
variants » XVII, $\mathrm{n}^{\circ} 1058$. On pourrait considérer EIAYPIONON comme une dittographie, mais dans ce cas le pronom ferait défaut, surtout car le participe est à l'accusatif ( ${ }^{\epsilon} \xi \in \lambda \theta o ́ \nu \tau \omega \nu \alpha \bigcup ่ \tau \hat{\omega} \nu$ $\mathrm{B} \aleph$ rell $\left.\left[-\alpha \cup \cup \tau \omega \nu f^{13} 69.565 .788 .1346\right]\right)$.

123 Erreur par échange consonantique $\left(\Xi>^{\mathrm{K}}\right)$ corrigée par la $p . m$.

${ }^{124}$ Par haplographie. Quant au sens symbolique sous-entendu dans le Codex de Bèze, voir « Les variants » XVII, $n^{\circ} 1069$.

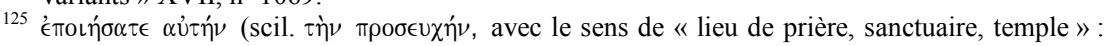
voir « Les variants » XVII, $\mathrm{n}^{\circ} 1072$.

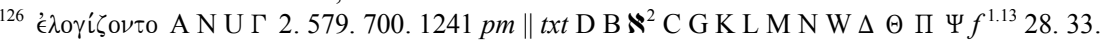
69. 118. 157. 565. 892. 1071. 1424. 2427. $2542 \mathrm{pm}$.

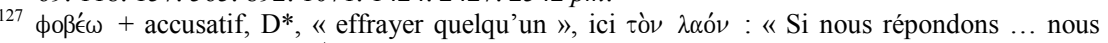
effrayons le peuple. " $\mathrm{D}^{\mathrm{A}}$ a corrigé suivant le parallèle de Mt 21,26. D'après Parker, Codex Bezae, 132, " $\mathrm{D}^{*}$ is simply a mistake for that (the active being obsolete in the Hellenistic period) ». Le caractère singulier de la leçon de $\mathrm{D}^{*}$, posée par Marc dans la bouche des dirigeants juives, confirme que c'est la bonne leçon.

128 Le scribe a confondu OIC avec la lettre onciale oméga $(\omega)$.

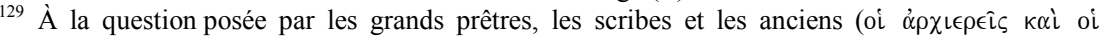

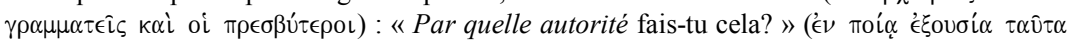

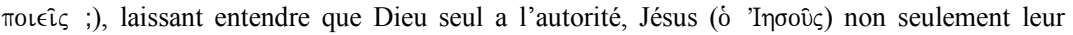
répond negativement, mais, en changeant la formule, leur laisse entendre que c'est lui même qui a l'autorité divine : "Moi non plus je ne vous dis pas avec quelle autorité je fais cela "

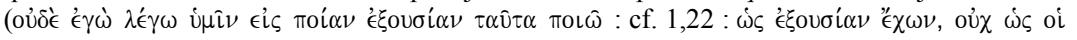

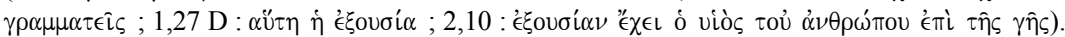




$\begin{array}{cc}12,1 & , / 12 \\ 12,4 & , / 23 \\ , & , / 24\end{array}$

$12,17329 \mathrm{~b} / 2$

$12,23 \quad " / 20$

$12,25 \quad$ " $/ 26$

$12,28330 \mathrm{~b} / 3$

$12,33 \quad " / 23$

$12,36331 \mathrm{~b} / 3$

" ",

$12,38 " / 10$

$12,42 \quad " / 20$

$12,43 \quad " / 26$

$13,1 \quad " / 33$

, $332 \mathrm{~b} / 1$

$13,4 \quad " / 14$

$13,5 \quad " / 16$

$13,10 \quad " / 30$

$13,16333 \mathrm{~b} / 20$

$13,31335 \mathrm{~b} / 2$
ANIIE $\Lambda \Omega N A$

EKEФA $\Lambda A I \Omega \Sigma A N$

HTIMHLAN

EOAYMAZONTO

H $\Gamma Y N H$

ANA $2 T H \Sigma O Y \Sigma I N$

$\mathrm{AYT} \Omega$

¿YNZHTOYNTQN

$\Omega \Sigma$ $\Sigma$ EAYTON

$\Theta \Omega \Sigma \Omega$

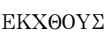

KAI T $\Omega N$ TE $\Lambda \Omega N \Omega N$

AMA

GAZOФYАAKION

ПО $\triangle \mathrm{A \Pi OI}$

IO $\triangle \mathrm{A}$ AI

OTAN ME $\Lambda$ EI

МН...П $\Lambda$ ANHЕEI

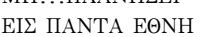

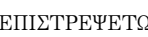

OY

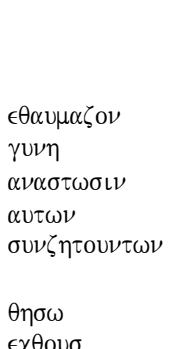

$\epsilon \chi \ominus$ ov

$\mu \iota \alpha$

$\gamma \alpha \zeta$ о $\nu \lambda \alpha \kappa\llcorner\mathrm{L \nu}$

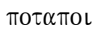

$\pi 0 \tau \alpha \pi \alpha \iota$

$\epsilon$

$\epsilon \iota \sigma$

$\epsilon \pi\llcorner\sigma \tau \rho \epsilon \psi \alpha \tau \omega$

ou $\mu \eta$

$\begin{array}{ll}\text { corr. } & \\ \text { b.1. } & \\ \text { cj. } & (v l \text { n.e. } \\ \mathrm{D}^{\text {s.m }} & \text { (B } v l \text { n.e. }) \\ \mathrm{D}^{\text {s.m }} & \text { B } v l \text { n.e. } \\ \mathrm{D}^{\mathrm{B}} & \mathrm{B} v l \text { n.e. } \\ \mathrm{D}^{\mathrm{A}} & \mathrm{B} v l \text { n.e. }\end{array}$

$\dot{\alpha} \mu \pi \epsilon \lambda \hat{\omega} \nu \alpha^{130}$

єК $€ \phi \alpha \lambda \alpha i \omega \sigma \alpha \nu$

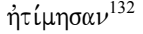

‘ $\theta \alpha \cup \mu \alpha \dot{\zeta} \zeta \mathrm{o \nu \tau o}$

ๆ $\gamma \cup \nu \eta^{133}$

$\mathrm{D}^{\mathrm{A}}$ ? $\quad \mathrm{B} v l$ n.e. $\quad \alpha \dot{v} \tau \hat{\omega}$

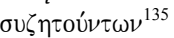

$\omega \varsigma \sigma \in \alpha \cup \tau o ́ \nu^{136}$

$\mathrm{D}^{\mathrm{A}} \quad \theta \eta \dot{\sigma} \sigma \omega$

$\mathrm{D}^{\text {s.m } 137} \quad \epsilon^{\prime} \chi \theta \rho o u ́ c$

corr. $\quad \kappa \alpha i \quad \tau \hat{\imath} \nu \quad \theta \in \lambda o ́ \nu-$

$\tau \omega \nu^{138}$

$\mathrm{D}^{\mathrm{A}}$ ? $\quad \mathrm{B} v l$ n.e. $\quad \ddot{\alpha} \mu \alpha^{139}$

$\mathrm{D}^{\mathrm{A}} \quad \gamma \alpha \zeta$ ○ $\nu \lambda \alpha \alpha_{\kappa}\left\llcorner\mathrm{\nu} \nu^{140}\right.$

$\mathrm{D}^{\mathrm{D}} \quad \mathrm{B} v l$ n.e. $\quad \pi \circ \delta \alpha \pi i^{141}$

$\mathrm{D}^{\mathrm{D}} \quad \mathrm{B} v l$ n.e. $\quad \pi \delta \delta \alpha \pi \mathrm{i}^{142}$

b.1. ${ }^{143} \quad v l$ n.e. $\quad$ ó $\tau \alpha \nu \mu \mu^{\prime} \lambda \lambda \in \mathrm{L}$

b.1. ${ }^{144} \quad v l$ n.e. $\quad \mu \eta \ldots \pi \lambda \alpha \nu \eta \dot{\sigma} \sigma \epsilon$

$\tau \alpha \mathrm{D}^{\mathrm{B}} \quad \mathrm{B} v l$ n.e.

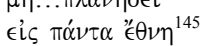

$\epsilon \pi\llcorner\sigma \tau \rho \epsilon \psi \alpha ́ \alpha \omega$

ou 146

${ }^{130}$ Cf. 12,2.8.9a.9b.

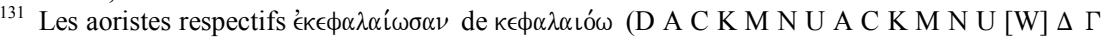
$\Theta \Pi f^{13}$ 2. 33. 157. 579. 1071. 1424. [1. 28. 118. 565. 700. 1582] Mi) et é $\kappa \epsilon \phi \alpha \lambda i ́ \omega \sigma \alpha \nu ~ d e$

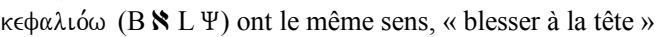

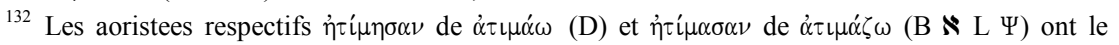
même sens, « mépriser, traiter avec dédain ».

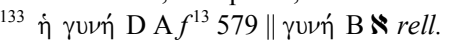

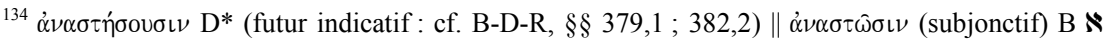
rell.

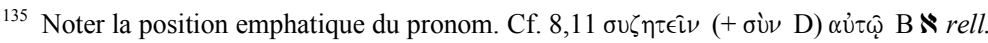

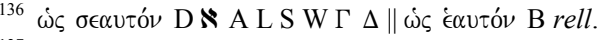

${ }^{137} \mathrm{D}^{\mathrm{s} . \mathrm{m} .}$ a eliminé le $\mathrm{K}$, mais sans y ajouter un $\mathrm{P}$.

138 et qui volunt $\mathrm{d}$.

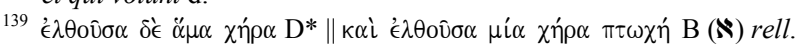

${ }^{140} \mathrm{La}$ lettre «G» dans GAZОФҮ $А$ АКION met en évidence que le scribe est un latin.

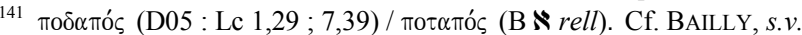

142 Voir n. précédente.

$143 \mu \mu^{\prime} \lambda \lambda \in \mathrm{L}$ (indicatif présent) D E M $\Gamma \Delta f^{13} 2.33 .69 .124^{*} .1071 \| \mu \mu^{\prime} \lambda \lambda \eta ̣$ (subjonctif présent) B $\aleph$ rell : URBÁN, «Intercambios vocálicos», 266.

$144 \pi \lambda \alpha \nu \eta ́ \sigma \epsilon$ (futur) D H G Y $f^{13} 2 *$. 28. $1071 \| \pi \lambda \alpha \nu \eta ́ \sigma \eta n$ (subjonctif) B $\aleph$ rell: URBÁN, «Intercambios vocálicos», 266.

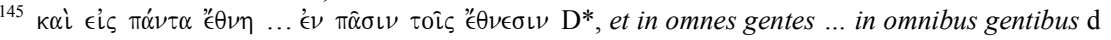

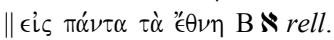




\begin{tabular}{|c|c|c|c|c|c|c|}
\hline 13,34 & $" / 13$ & $\Theta Y P O Y P \Omega$ & $\theta u \rho \omega \rho \omega$ & $\mathrm{D}^{\mathrm{B}}$ & & 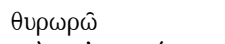 \\
\hline 14,1 & 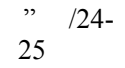 & $\begin{array}{l}\text { KAI | AПOKTEI- } \\
\text { N } \Omega \text { IIN }\end{array}$ & $\alpha \pi O \kappa \tau \epsilon \iota \nu \omega \sigma \iota \nu$ & $\mathrm{D}^{\mathrm{s.m} .}$ & B $v l$ n.e. & 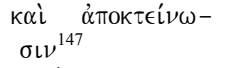 \\
\hline 14,3 & $" / 29$ & ГҮГН & $\gamma \cup \nu \eta$ & $\mathrm{D}^{\mathrm{B}}$ & & 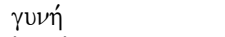 \\
\hline 14,5 & $336 \mathrm{~b} / 4$ & EN AYTH & $\alpha \cup \tau \eta \nu^{148}$ & $\mathrm{D}^{\mathrm{s.m} .}$ & B $v l$ n.e. & 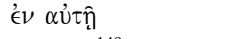 \\
\hline 14,13 & $" / 31$ & ҮПАГЕ & $\mathrm{v} \pi \alpha \gamma \epsilon \tau \epsilon$ & $\mathrm{D}^{\mathrm{B}}$ & & $\dot{u} \pi \alpha^{\prime} \gamma \epsilon \tau \epsilon^{149}$ \\
\hline 14,16 & $337 b / 7-8$ & 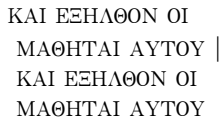 & 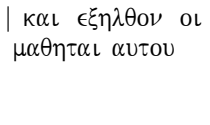 & $\mathrm{D}^{\text {s.m. }} 150$ & & 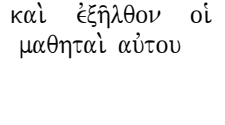 \\
\hline 14,20 & $" / 21$ & TPYBA $\Lambda$ ION & $\tau \rho \cup \beta \lambda\llcorner\mathrm{\iota \nu}$ & $\mathrm{D}^{\mathrm{s.m} .}$ & & $\tau \rho u ́ \beta \lambda \iota 0 \nu$ \\
\hline 14,24 & $" / 33$ & TO TH $\Sigma \Delta \mathrm{IA} \Theta H K H \Sigma$ & $\tau \eta \sigma \delta\llcorner\alpha \theta \eta \kappa \eta \sigma$ & $\mathrm{D}^{\text {s.m. }}$ & B & ${ }_{\kappa \eta \varsigma^{151}}^{\tau \eta \hat{~}}$ \\
\hline$"$ & $338 \mathrm{~b} / 1$ & EXXYNNOMENON & & corr. & & 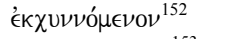 \\
\hline 14,27 & $" / 8$ & $\Sigma \mathrm{KAN} \Delta \mathrm{A} \Lambda \mathrm{I} \Sigma \mathrm{A} \Sigma \Theta \mathrm{AI}$ & & corr. & & $\sigma \kappa \alpha \nu \delta \alpha \lambda i ́ \sigma \epsilon \sigma \theta \epsilon^{153}$ \\
\hline 14,29 & $" / 15$ & $\begin{array}{l}\text { А } \Lambda \Lambda \text { OYK EГ } \Omega \text { OY } \\
\Sigma \mathrm{KAN} \triangle \mathrm{A} \Lambda \mathrm{I} \Sigma \mathrm{H}- \\
\Sigma \mathrm{OMAI}\end{array}$ & $\begin{array}{l}\alpha \lambda \lambda \quad \text { ouk } \epsilon \gamma \omega \\
\sigma \kappa \alpha \nu \delta \alpha \lambda \iota \sigma \theta \eta- \\
\sigma o \mu \alpha \iota\end{array}$ & $\mathrm{D}^{\text {s.m. }}$ & B $v l$ n.e. & 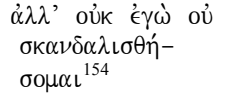 \\
\hline 14,31 & $" / 20$ & EAN MH $\Delta \mathrm{EH}$ & $\begin{array}{l}\epsilon \alpha \nu \quad \delta \epsilon \eta \mu \epsilon \\
\epsilon \alpha \nu \mu \epsilon \delta \epsilon \eta\end{array}$ & $\begin{array}{l}\mathrm{D}^{\mathrm{B}} \\
\text { corr. }\end{array}$ & & $\epsilon^{\prime} \alpha \dot{\alpha} \nu \mu \epsilon \delta^{\prime} \mathfrak{\epsilon}^{155}$ \\
\hline 14,32 & $" / 24$ & ГHЕAMANEI & & corr. & & $\Gamma \epsilon \theta \sigma \eta \mu \alpha \nu i^{156}$ \\
\hline 14,33 & $" / 29$ & AKH $\triangle$ EMONEIN & $\alpha \delta \eta \mu о \nu \in L \nu$ & $\mathrm{D}^{\mathrm{A}}$ & B $v l$ n.e. & 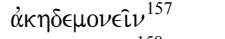 \\
\hline 14,41 & $339 \mathrm{~b} / 20$ & ANAПAE $\Sigma \Theta A I$ & & corr. & & $\dot{\alpha} \nu \alpha \pi \alpha \dot{\epsilon} \epsilon \sigma \theta \epsilon^{158}$ \\
\hline
\end{tabular}

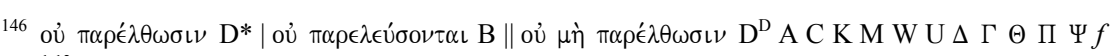

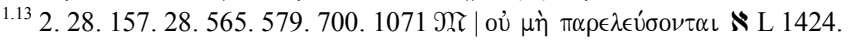

${ }^{147}$ Participe $+\kappa \alpha i ́$ adverbiale (séparé par un espace en blanc et placé à la fin du stique) + verbe personnel est une caractéristique du Codex de Bèze.

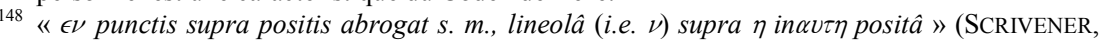
BEZAE CODEX CANTABRIGIENSIS, 439, col. 2). Seulement $\mathrm{D}^{\mathrm{s.m} .}$ présente la leçon AYTHN, D* lit

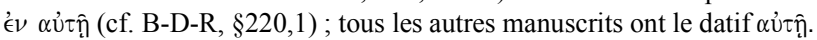

149 Par haplographie.

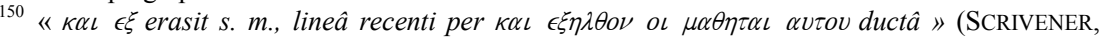
BEZAE CODEX CANTABRIGIENSIS, 439, col. 2). Répétition du stique par inadvertance du scribe.

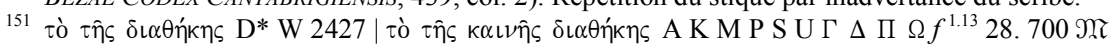
lat sy sa ${ }^{\mathrm{mss}} \mathrm{bo}^{\mathrm{pt}} \| \tau \hat{\eta} \varsigma \delta\left\llcorner\alpha \theta \eta \dot{\kappa} \eta \varsigma \mathrm{B} \aleph \mathrm{CD}^{\mathrm{s.m} .} \mathrm{L} \Theta \Psi 563 \mathrm{k} \mathrm{sa}^{\mathrm{mss}}\right.$ bo $^{\mathrm{pt}}$.

152 Par assimilation.

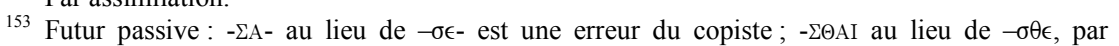
échange vocalique.

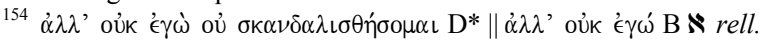

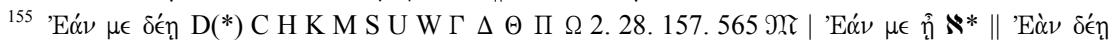
$\mu \epsilon \mathrm{B} \aleph^{2} \mathrm{~A} \mathrm{D}^{\mathrm{B}} \mathrm{L} \mathrm{N} \Psi 083 f^{13} 2^{\mathrm{c}} .579 .700 .892 .1071 .1118 .1424 .1582 .2427 .2542$ al. $\mathrm{D}^{*}$ lit erronément $\mathrm{MH} ; \mathrm{D}^{\mathrm{B}}$ lit ME mais en harmonisant avec le texte alexandrin.

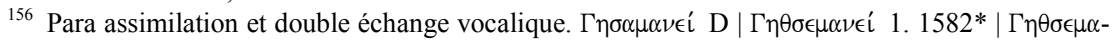

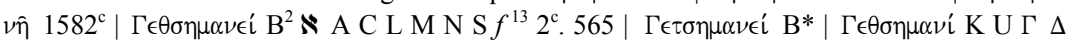

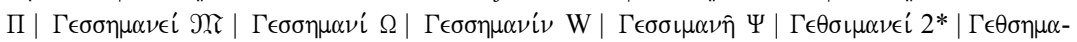
$\nu \eta ́$ $\Theta$ 118. 157. 579. 700. 1071. 1424. Grande confusion dans la transcription du terme hébreu.

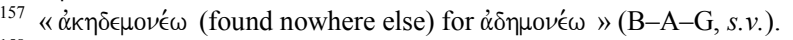

${ }^{158}$ Par haplographie. 


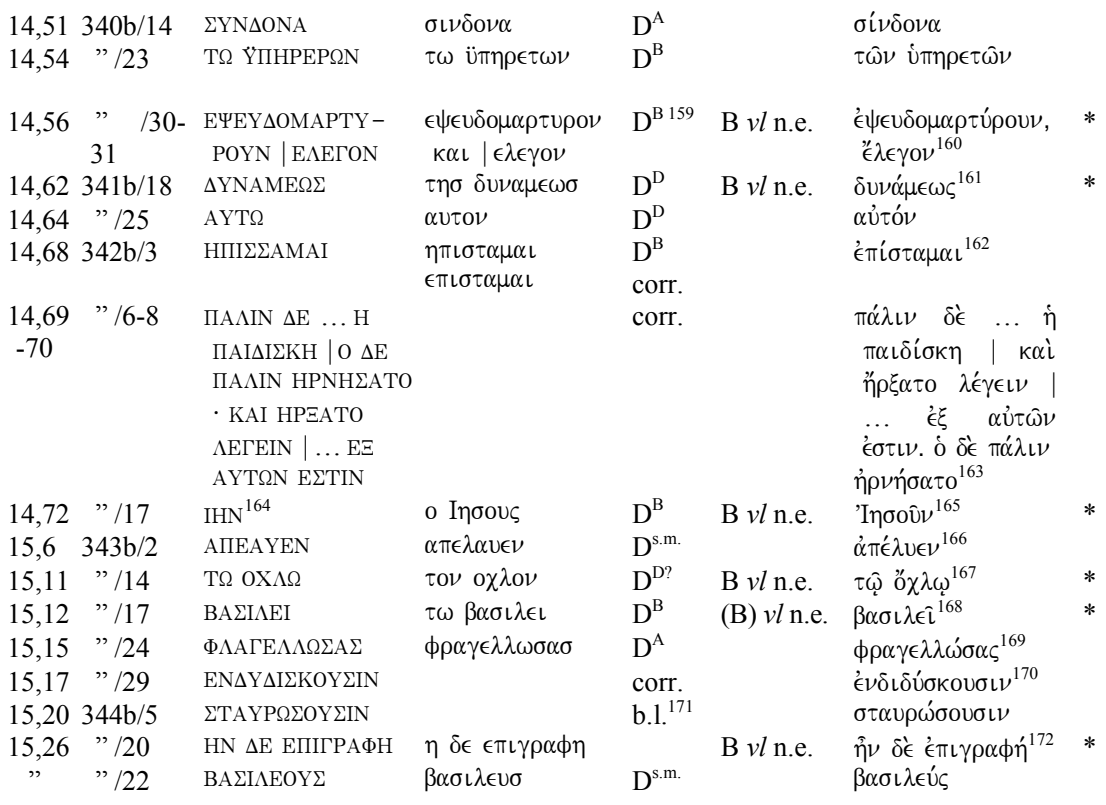

159 Voir Parker, Codex Bezae, 148.

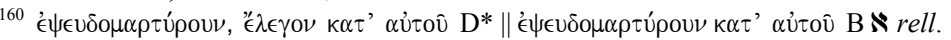

$161 \delta \cup \nu \alpha \dot{\mu} \mu \epsilon \varsigma \mathrm{D} * \| \tau \hat{\jmath} \varsigma \delta u \nu \alpha ́ \mu \epsilon \omega \varsigma \mathrm{B} \aleph$ rell.

162 Par échange de $\mathrm{H}$ au lieu de $\mathrm{E}$ et par assimilation consonantique.

163 L'inversion des incises est antérieur à l'élaboration du Codex de Bèze, pusqu'il se trouve aussi dans la page latine : cum vidisset eum ancilla $\mid$ at ille rursus negavit $\cdot$ et coepit dicere ... ex ipsis est.

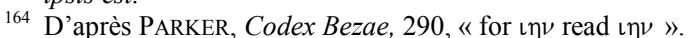

165 Voir mon article «"TO PHMA O EIPEN IHN”, ¿Un error del copista del Còdex Bezae o la lliçó original de Mc 14,72? », RCatT 31 (2006) 429-438.

166 Par haplographie.

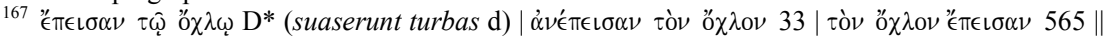

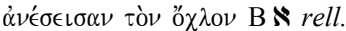

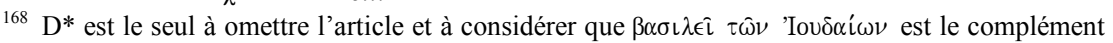

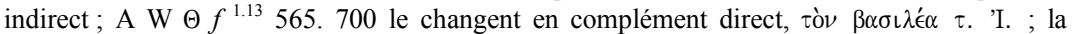

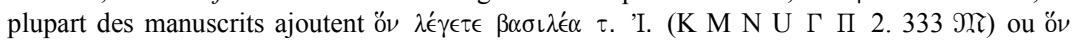

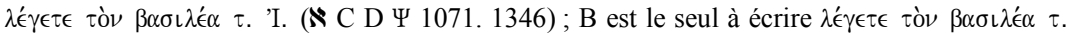
'I.

169 La forme $Ф \Lambda \mathrm{AГЕ} \Lambda \Lambda \Omega \mathrm{A} \Sigma$ est dûe probablement à l'influence du latin flagellare.

${ }_{170}$ Par metathèse $-\Delta \mathrm{Y} \Delta \mathrm{I}->-\Delta \mathrm{I} \Delta \mathrm{Y}-$.

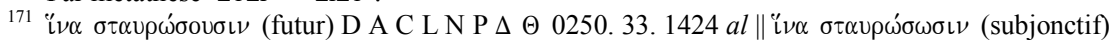

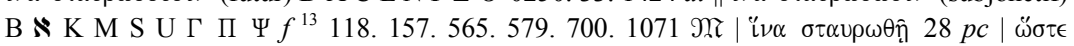
$\sigma \tau \alpha \cup \rho \omega \hat{\sigma} \sigma \iota\left\llcorner f^{1} 2542^{\mathrm{s}}\right.$

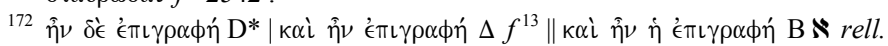




\begin{tabular}{|c|c|c|c|c|c|c|}
\hline 15,27 & $" / 23$ & $\Lambda \mathrm{H} \Sigma \mathrm{TAI}$ & $\lambda \eta \sigma \tau \alpha \sigma$ & $\mathrm{D}^{\mathrm{A}}$ & B $v l$ n.e. & $\lambda \eta \sigma \tau \alpha i$ \\
\hline 15,34 & $345 \mathrm{~b} / 8$ & \multicolumn{2}{|l|}{$\begin{array}{l}\text { H } \Lambda \text { EI H } \Lambda \text { EI } \Lambda \text { AMA } \\
\text { ZA } \Theta A N E I\end{array}$} & \multicolumn{2}{|l|}{ b.1. $?^{173}$} & $\begin{array}{l}\epsilon \lambda \iota \in \lambda \iota \quad \lambda \alpha \mu \alpha \zeta \alpha- \\
\phi \theta \alpha \nu \iota\end{array}$ \\
\hline 15,36 & $" / 13$ & $\Sigma \Phi O N Г O N$ & & \multicolumn{2}{|l|}{ corr. } & 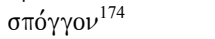 \\
\hline$"$ & $" / 14$ & 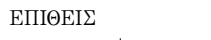 & & \multirow{2}{*}{\multicolumn{2}{|c|}{ corr. }} & $\epsilon \pi \iota \theta \epsilon i \varsigma \quad \kappa \alpha \lambda \alpha \dot{\alpha} \mu \omega$ \\
\hline & & $\operatorname{KA} \Lambda \mathrm{AM} \Omega \mid$ & & & & 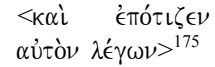 \\
\hline 15,43 & $" / 32$ & 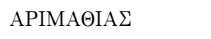 & & \multicolumn{2}{|l|}{ b.1. ${ }^{176}$} & 'A $\rho \iota \alpha \theta^{\prime} i \alpha \varsigma$ \\
\hline " & $346 b / 3$ & ЕTHгATO & $\eta \tau \eta \sigma \alpha \tau 0$ & \multicolumn{2}{|l|}{$\mathrm{D}^{\mathrm{B} 177}$} & $\eta \emptyset \tau \eta ́ \sigma \alpha \tau o$ \\
\hline 15,44 & $" / 4$ & EITE@NHKEI & $\tau \in \theta \nu \eta \kappa \in \iota$ & \multicolumn{2}{|l|}{$\mathrm{D}^{\mathrm{s.m} .}$} & $\tau \in \theta \nu \eta ́ \kappa \in \iota^{178}$ \\
\hline 16,3 & $" / 23$ & HMION & $\eta \mu \iota \nu$ & \multicolumn{2}{|l|}{$\mathrm{D}^{\mathrm{p} . \mathrm{m} .}$} & $\dot{\eta} \mu \imath \nu$ \\
\hline 16,6 & $" / 33$ & $\mathrm{H}$ & $\mu \eta^{179}$ & \multicolumn{2}{|l|}{ Yoder } & Mń \\
\hline 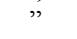 & $347 b / 2$ & TOION AYTOY & 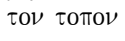 & $\mathrm{D}^{\mathrm{C}}$ & (B $v l$ n.e.) & 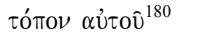 \\
\hline
\end{tabular}

J'ai pu identifier un nombre total de 205 possibles erreurs dans la première main de D05. Parmi elles on doit en écarter 92 qui ne sont pas des erreurs réelles : de nombreuses corrections sont dûes à des harmonisations avec le type de texte qui était familier aux correcteurs $(65 \times)$; certaines figuraient déjà dans la première main $($ p.m. $3 \times)$ ou bien elles étaient tout à fait inutiles, corrections qui en général n'ont pas été enregistrées dans le ${ }^{27} \mathrm{~N}-\mathrm{A}(79 \times)$. Le plus grand nombre de corrections que j'ai approuvées (parmi lesquelles il y a 45 corrections propres) sont des erreurs par assimilation, dissimilation, métathèse, dittographie, haplographie ; d'autres sont dûes à des échanges consonantiques par dissimilation progressive ou regressive ou à l'influence de la page latine. Je

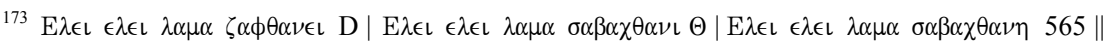

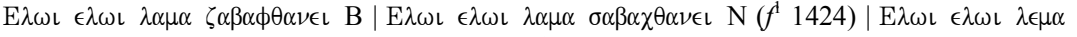

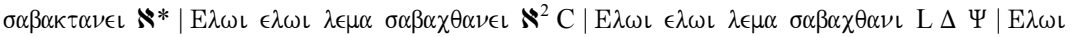
$\epsilon \lambda \omega \iota \quad \lambda \iota \mu \alpha \quad \sigma \alpha \beta \alpha \chi \theta \alpha \nu \iota$ K M N U Г П $f^{13} 33.700 \mid$ E $\lambda \omega \iota \in \lambda \omega \iota \quad \lambda \iota \mu \alpha \sigma \alpha \beta \alpha \chi \nu \alpha \nu \iota$ P (118) |E E

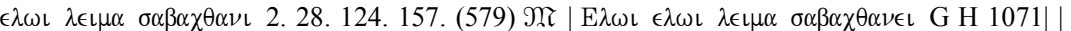
E $\lambda \omega \iota \in \lambda \omega \iota \quad \lambda \iota \mu \alpha \sigma \iota \beta \alpha \kappa \theta \alpha \nu \in \iota$ A. Grande confusion dans la transcription de l'hébreu. D05 traduit

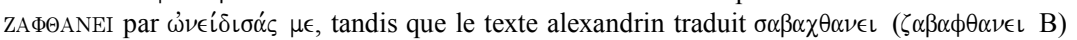

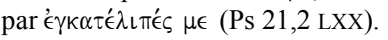

174 Par assimilation.

${ }^{175}$ L'incise omis par le scribe dans la page grecque figurait sans doute dans l'original, ce

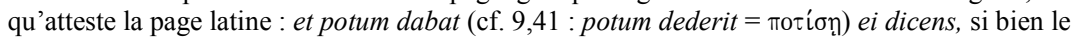
même scribe dans la page latine a oublié de traduire $\epsilon \pi \iota \theta \epsilon i \varsigma \kappa \alpha \lambda \alpha^{\prime} \mu \omega$.

176 'A $\rho \mu \alpha \theta i \alpha \varsigma$ D, Arimathia d $\aleph^{2}$ 69. 124. 1071. 1346. 2427 pc lat : cf. Lc 23,51 D \| 'A $\rho \mu \alpha \theta \alpha i \alpha \varsigma$ $\mathrm{B} \aleph *$ rell.

177 «... this correction is by B » (PARKER, Codex Bezae, 296).

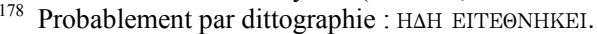

179 Cf. PARKer, Codex Bezae, $290:$ : for $\eta$ read $\mu \eta$ (Yoder) ».

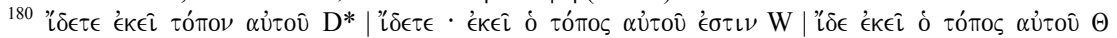

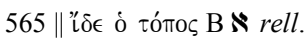


tiens compte des erreurs réelles identifiées et décrites dans les Tables 1 et 2 pour les rectifier dans l'édition imprimée de l'Évangile de Marc selon le Codex de Bèze, en préparation.

\section{Délimitation des séquences}

Une deuxième remarque concerne la délimitation précise des séquences (à toute vraisemblance, des unités de prédication) qui donnent la structure à cet évangile. Il existe des désaccords assez importants entre les éditeurs et les exégètes en ce qui concerne la division des séquences et la longueur exacte de chacune d'elles. Il était essentiel pour mener à bien l'identification du niveau rédactionnel de chaque séquence, de les délimiter avec précision. Pour cela, j'ai utilisé quatre critères, dont au moins un critère devait être présent pour pouvoir parler de séquence indépendant :

Précision de temps, explicite ou implicite

Précision de lieu dans l'en-tête d'une séquence

Entrée ou participation dans la scène d'un nouveau personnage

Changement de thème

Une fois appliqués ces critères, j'ai pu distinguer clairement 98 séquences que je numéroterai successivement dans mon édition de l'Évangile de Marc selon le système présenté dans la Table 3 . On trouvera dans cette table quatre séquences marquées avec des parenthèses angulaires $(<>)$ qui ne figurent pas dans le texte canonique de Marc, mais que je me suis permis d'y ajouter, convaincu qu'elles figuraient dans la deuxième rédaction de cet évangile : ce sont les trois séquences conservées dans l'Évangile Secret de Marc (Séqs. 6061 et 63$)^{181}$ et la séquence sur la femme adultère préservée par le Codex de Bèze dans l'Évangile de Jean en son état actuel (Séq. 70 : Jn 7,53-8,11 D05). ${ }^{182}$

\section{Phénomènes qui ont suscité l'hypothèse}

\subsection{Nom ou pronom?}

Ces clarifications faites, je propose de passer en revue les phénomènes qui m'ont porté à formuler mon hypothèse, c'est-à-dire, qu'il existe deux, ou même trois, niveaux rédactionnels dans l'Évangile de Marc, chacun l'oeuvre de l'auteur lui-même. Le premier phénomène est très simple : j'ai constaté que

${ }^{181}$ Morton SMITH, Clement of Alexandria and a Secret Gospel of Mark, Cambridge MA : Harward University Press, 1973, et The Secret Gospel : the Discovery and Interpretation of the Secret Gospel According to Mark, New York : Harper, 1973. Dans l'édition que je prépare j'exposerai les raisons de poids qui m'ont incliné à les considérer comme authentiques.

182 Josep RIUS-CAMPS, «The Pericope of the Adulteress Reconsidered : The Nomadic Misfortunes of a Bold Pericope », New Testament Studies 53 (2007), pp. 379-405. 
dans à peu près la moitié des séquences s'explicite le nom de Jésus, alors que dans l'autre moitié apparait le pronom seul ( $\alpha$ úós, dans les divers cas de la déclinaison), l'article pronominal (ó $\delta \dot{e})$ ou simplement le verbe en temps personnel $^{183}$.

Selon l'analyse que j'expliquerai dans la prochaine section (3.2), les séquences où le nom de Jésus est absent seraient de la première rédaction, et celles où il est présent appartiendraient à une rédaction ultérieure ; enfin quelques séquences auraient été ajoutées par la suite dans une troisième rédaction.

Dans la table ci-jointe je donne toutes les fréquences du nom de Jésus qui se trouvent dans le Codex de Bèze et le Codex Vaticanus (le symbol $\varnothing$ indique son absence dans l'un ou l'autre codex). Dans la première colonne figure le numéro de la séquence, avec un, deux ou trois astérisques pour indiquer la rédaction à laquelle elle appartient suivant le Codex de Bèze; dans la deuxième, le chapître et les versets ; dans la troisième, le numéro du folio du Codex de Bèze et la ligne correspondante ; dans la quatrième, l'attestation ou

183 Je ne tiens pas compte de la mention de Jésus dans les deux séquences initiales : Séq. 1 (1,1-

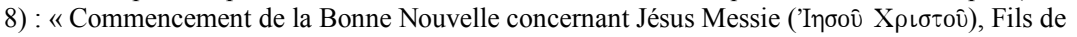
Dieu, comme il est écrit dans Isaïe, le prophête » $(1,1$ D05); Séq. 2 (1,9-11) : «Il est arrivé en

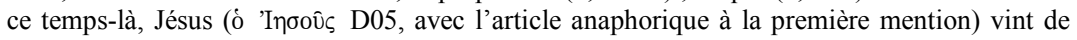
Nazareth de Galilée et il fut baptisé par Jean dans le Jourdain » (1,9 D05).

Je mets de côté également les passages où le nom est mentionné en discours direct à l'intérieur d'une séquence: Séq. 6 (1,21-28) : "Que nous veux tu, Jésus le Nazarénien? » $(1,24)$; Séq. 25 (5,1-10) : «Que me veux-tu, Jésus, fils du Dieu Très Haut? » $(5,6)$; Séq. 64 $(10,46-52)$ : «Quand il apprit : “C'est Jésus, le Nazarénien ”, il se mit à crier : " Fils de David, Jésus, aie pitié de moi! ”» (10,47 D05) ; Séq. 90 (14,66-72) : «Voyant Pierre qui se chauffait, elle [une servante] le dévisagea et dit : " Toi aussi tu étais avec Jésus, le Nazarénien " » $(14,67$ D05) ; Séq. 98 (16,2-8) : « Ne vous effrayez pas. C'est Jésus que vous cherchez, le crucifié? Il est ressuscité » $(16,6 \mathrm{D} 05)$.

Il va de même pour les endroits vers la fin de l'évangile où le nom était indispensable pour éviter des confusions avec d'autres personnages nommés dans le contexte précédent : Séq. 84 (14,26-31) : « Mais Pierre lui dit ... Et lui dit Jésus ... » (14,29-30) ; Séq. 89 (14,53-65) : « Et ils emmenèrent Jésus chez le Grand Prêtre ... [Dans la séquence précédent il s'agissait d'un jeune homme qui s'enfuit tout nu (14,51-52)]; Pierre l'avait suivi de loin ... Or, les grands prêtres et tout le Sanhédrin cherchaient un témoignage contre Jésus » (14,54-55); Séq. 90 $(14,66-72)$ : «Et aussitôt, pour le seconde fois, un coq chanta. Alors Pierre se rappella de la parole avec laquelle il avait répliqué à Jésus. Et il commença à pleurer » (14,72 D05); Séq. 91 $(15,1-5)$ : «Et aussitôt, le matin [immédiatement après les négations de Pierre], les grands prêtres se préparèrent à se réunir ... tout le Sanhédrin, et après avoir ligoté Jésus, ils l'emmenèrent en haut à la résidence («ils l'emmenèrent», B03 ) et ils le livrèrent à Pilate » (15,1 D05); «Pilate l'interrogea de nouveau ... Mais Jésus ne répondit plus rien, si bien que Pilate était étonné » (15,4-5) ; Séq. 92 (15,6-15) : «Pilate alors leur relâcha Barabbas; après avoir fait flageller Jésus, il le livra pour être crucifié » (15,15 D05); Séq. 95 (15,33-41) : "Quelqu'un courut, ayant rempli une éponge du vinaigre et, l'ayant mise au bout d'un roseau, il lui donnait à boire en disant : “Attendez, voyons si Élie va venir le descendre ! ” Or Jésus, jettant un grand cri, expira » (15,36 D05); Séq. 96 (15,42-46) : « Joseph d'Arimathie ... ayant pris courage il vint auprès de Pilate et demanda le cadavre de Jésus » (15,43 D05). 
l'absence du nom de Jésus dans le Codex de Bèze; dans la cinquième, son attestation ou son absence dans le Codex Vaticanus; dans la dernière colonne j'enumère les pronoms utilisés par le narrateur dans le Codex de Bèze.

Table 3

Absence ou présence du nom de Jésus

\begin{tabular}{|c|c|c|c|c|c|}
\hline Séquence & Chap. /v. & Fol. / l. & D05 & $B 03$ & 8,5 \\
\hline${ }^{*} 1$ & $1,1-8$ & $285 \mathrm{~b}, 1$ & 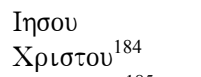 & I & \\
\hline${ }^{*} 2$ & $1,9-11$ & $285 \mathrm{~b}, 24$ & o Inбous ${ }^{185}$ & Inoous & $\epsilon L \varsigma \alpha \cup \tau O \nu$ \\
\hline$* 3$ & $1,12-13$ & & $\varnothing$ & $\varnothing$ & $\alpha \cup \tau 0 \nu,-\omega$ \\
\hline${ }^{* *} 4$ & $1,14-15$ & 286,3 & o Inбous & 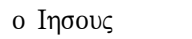 & \\
\hline **5 5 & $1,16-20$ & $286 b, 12$ & o Inбous & o Inбous & $\alpha \cup \tau \omega,-\omega$ \\
\hline${ }^{*} 6$ & $1,21-28$ & $\begin{array}{l}286 b, 29 \\
286 b, 32\end{array}$ & 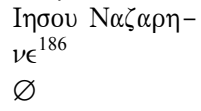 & 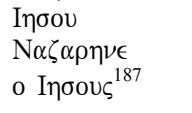 & $\alpha \cup \tau O U,-\omega,-O U$ \\
\hline${ }^{*} 7$ & $1,29-31$ & & $\varnothing$ & $\varnothing$ & $\alpha U \tau \omega$ \\
\hline *8 & $1,32-34$ & & $\varnothing$ & $\varnothing$ & $\begin{array}{l}\pi \rho \circ \varsigma \alpha \cup \tau o \nu,-o v \\
-\mathrm{o \nu}\end{array}$ \\
\hline${ }^{*} 9$ & $1,35-39$ & & $\varnothing$ & $\varnothing$ & $\alpha \cup \tau 0 \nu,-0 \nu,-\omega$ \\
\hline${ }^{*} 10$ & $1,40-45$ & & $\varnothing$ & $\varnothing$ & $\begin{array}{l}\pi \rho \circ \varsigma \alpha \cup \tau 0 \nu,-0 \nu \\
-0 \nu, \pi \rho \circ \varsigma-0 \nu\end{array}$ \\
\hline${ }^{* *} 11$ & $2,1-12$ & $\begin{array}{l}288 \mathrm{~b}, 28 \\
288 \mathrm{~b}, 31 \\
289 \mathrm{~b}, 6\end{array}$ & 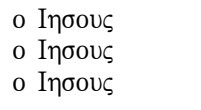 & 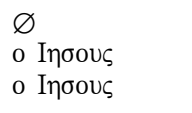 & $\pi \rho \circ \varsigma \alpha u \tau o \nu$ \\
\hline${ }^{*} 12$ & $2,13-14$ & & $\varnothing$ & $\varnothing$ & $\pi \rho \circ \varsigma \alpha \cup \tau 0 \nu,-\omega$ \\
\hline${ }^{* *} 13$ & $2,15-17$ & $\begin{array}{l}289 b, 30 \\
290 b, 5\end{array}$ & 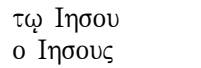 & 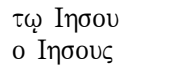 & $\begin{array}{l}\alpha \cup \tau o v,-o v,-\omega, \\
-o v\end{array}$ \\
\hline${ }^{*} 14$ & $2,18-22$ & $290 \mathrm{~b}, 14$ & $\varnothing$ & 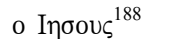 & $\alpha \cup \tau \omega$ \\
\hline${ }^{*} 15$ & $2,23-28$ & & $\varnothing$ & $\varnothing$ & $\alpha \cup \tau o \nu$ \\
\hline${ }^{* * *} 16$ & $3,1-7 \mathrm{a}$ & $291 b, 32$ & o $\delta \epsilon$ I $\eta \sigma o u \varsigma$ & 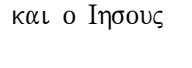 & $\begin{array}{l}\alpha \cup \tau \mathrm{O} \nu,-\mathrm{o \nu}, \mathrm{\kappa} \alpha \tau{ }^{\prime} \\
-\mathrm{ov},-\mathrm{o \nu},-\mathrm{o \nu}\end{array}$ \\
\hline${ }^{*} 17$ & $3,7 b-12$ & & $\varnothing$ & $\varnothing$ & $\begin{array}{l}\pi \rho \varsigma \alpha \cup \tau o \nu,-0 \nu, \\
-\omega,-0 \nu, \epsilon \nu-\omega, \\
-o v,-0 \nu,-\omega, \\
-o \nu\end{array}$ \\
\hline${ }^{*} 18$ & $3,13-19$ & & $\varnothing$ & $\varnothing$ & $\begin{array}{l}\alpha \cup \tau 0 \varsigma, \pi \rho \circ \varsigma-\mathrm{o \nu}, \\
\mu \in \tau^{\prime}-\mathrm{ov},-\mathrm{o \nu}\end{array}$ \\
\hline${ }^{*} 19$ & $3,20-21$ & & $\varnothing$ & $\varnothing$ & $\pi \in \rho \iota \alpha \cup \tau o u,-O \nu$ \\
\hline
\end{tabular}

184 Première mention dans l'évangile.

185 Première mention après la séquence concernant Jean le baptiste.

186 Discours direct.

187 Selon B03 cette séquence ne pourrait pas être attribuée à la première rédaction.

188 Selon B03 cette séquence ne pourrait pas être attribuée à la première rédaction. 


\begin{tabular}{|c|c|c|c|c|c|}
\hline ** 20 & $3,22-30$ & $293 b, 12$ & 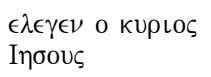 & $\begin{array}{l}\varnothing^{189}(\epsilon \lambda \epsilon \gamma \in \nu \\
\alpha \cup \tau \operatorname{L} \varsigma)\end{array}$ & \\
\hline *21 & $3,31-35$ & & $\varnothing$ & $\varnothing$ & $\begin{array}{l}\alpha \cup \tau O v,-o v, \pi \rho \circ \varsigma \\
-0 \nu,-0 \nu,-\omega\end{array}$ \\
\hline${ }^{*} 22^{\mathrm{a}}$ & $4,1-9$ & & $\varnothing$ & $\varnothing$ & 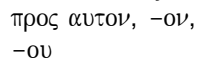 \\
\hline${ }^{* * *} 23^{190}$ & $\begin{array}{l}4,10- \\
25.34\end{array}$ & & $\varnothing$ & $\varnothing$ & $\begin{array}{l}\alpha \cup \tau o \nu,-o v \mid \\
-o v\end{array}$ \\
\hline${ }^{*} 22 \mathrm{~B}$ & $4,26-33$ & & $\varnothing$ & $\varnothing$ & \\
\hline${ }^{*} 24$ & $4,35-41$ & & $\varnothing$ & $\varnothing$ & $\begin{array}{l}\alpha \cup \tau o \nu, \mu \in \tau,-o v, \\
-o \zeta,-0 \nu,-\omega\end{array}$ \\
\hline ***25 & $5,1-10$ & $\begin{array}{l}298 b, 16 \\
298 b, 19 \\
298 b .21\end{array}$ & 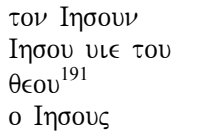 & 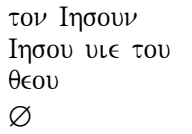 & $\alpha \cup \tau \omega,-\omega,-0 \nu$ \\
\hline ***26 & $5,11-17$ & $\begin{array}{l}298 b, 31 \\
299 b, 7\end{array}$ & 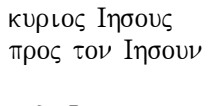 & 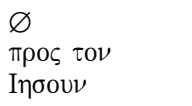 & $\alpha \cup \tau 0 \nu,-0 \nu$ \\
\hline **27 & $5,18-20$ & $\begin{array}{l}299 b, 18 \\
299 b, 23\end{array}$ & 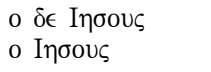 & $\begin{array}{l}\varnothing \\
\text { o Inoous }\end{array}$ & $\begin{array}{l}\alpha u \tau o v,-o \nu, \mu \in \tau, \\
-o u\end{array}$ \\
\hline${ }^{* *} 28$ & $5,21-24$ & $299 b, 24$ & $\tau O u$ I & $\tau O U$ I $\eta \sigma O U$ & 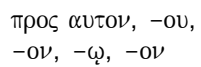 \\
\hline${ }^{* *} 29$ & $5,25-34$ & $\begin{array}{l}300 \mathrm{~b}, 6 \\
300 \mathrm{~b}, 14 \\
300 \mathrm{~b}, 26\end{array}$ & 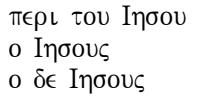 & 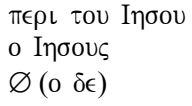 & $\begin{array}{l}\alpha \cup \tau o v, \alpha \pi^{\prime}-\mathrm{ov}, \\
-\mathrm{ov},-\omega,-\omega, \\
-\omega\end{array}$ \\
\hline${ }^{* *} 30$ & $5,35-43$ & $300 \mathrm{~b}, 33$ & o $\delta \epsilon$ I $\eta \sigma o u \varsigma$ & o $\delta \epsilon$ I $\eta \sigma o u \varsigma$ & $\begin{array}{l}\alpha \cup \tau o v,-\omega,-\omega, \\
-o v,-o \zeta, \mu \in \tau \\
-o v\end{array}$ \\
\hline ***31 & $6,1-6 a$ & $302 b, 6$ & o Inбous & 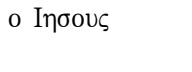 & $\begin{array}{l}\alpha \cup \tau o v,-\omega,-o v, \\
-o v, \epsilon \nu-\omega\end{array}$ \\
\hline *32 & $6,6 b-13$ & & $\varnothing$ & $\varnothing$ & \\
\hline *33 & $6,14-15$ & & $\varnothing$ & $\varnothing$ & $\alpha \cup \tau o u$ \\
\hline${ }^{* * *} 34^{192}$ & $6,15-29$ & & $\varnothing$ & $\varnothing$ & \\
\hline${ }^{* *} 35$ & $6,30-31$ & $304 b, 26$ & 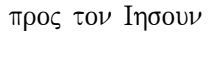 & 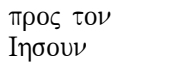 & $\alpha \cup \tau \omega$ \\
\hline **36 & $6,32-34$ & $\begin{array}{l}304 b, 29 \\
305 b, 7\end{array}$ & 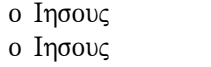 & $\begin{array}{l}\varnothing \\
\varnothing^{193}\end{array}$ & \\
\hline$* * 37$ & $6,35-46$ & $\begin{array}{l}305 b, 17 \\
305 b, 22 \\
305 b, 24\end{array}$ & 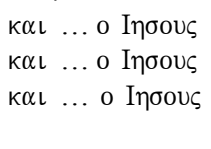 & $\begin{array}{l}\varnothing^{194}\left(\begin{array}{ll}0 & \delta \epsilon\end{array}\right) \\
\varnothing\left(\begin{array}{ll}0 & \delta \epsilon\end{array}\right) \\
\varnothing(\kappa \alpha \iota \quad \ldots \\
\alpha u \tau O \iota \varsigma)\end{array}$ & $\begin{array}{l}\alpha \cup \tau o v,-\omega,-\omega \\
-\omega,-O U,-o v \\
-o \nu,-o \zeta\end{array}$ \\
\hline
\end{tabular}

189 Selon B03 cette séquence aurait dû être attribuée à la première rédaction.

190 Explication de la parabole du semeur aux disciples à part de la foule.

191 Discours direct.

192 Longue amplification sur Hérode et Jean-Baptiste.

193 Selon B03 cette séquence aurait dû être attribuée à la première rédaction. 
Josep Rius-Camps

\begin{tabular}{|c|c|c|c|c|c|}
\hline **38 & $6,47-52$ & $306 b, 17$ & o Inбous & $\varnothing^{195}$ & $\alpha \cup \tau O \varsigma,-O \nu$ \\
\hline **39 & $6,53-56$ & $307 b, 5$ & 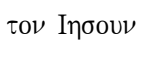 & $\varnothing^{196}$ & 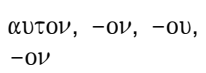 \\
\hline${ }^{*} 40$ & $7,1-16$ & & $\varnothing$ & $\varnothing$ & $\begin{array}{l}\pi \rho \circ \varsigma \alpha v \tau o \nu,-o v \\
-0 \nu\end{array}$ \\
\hline *** $41^{197}$ & $7,17-23$ & & $\varnothing$ & $\varnothing$ & $\alpha u \tau o \nu,-o u$ \\
\hline$* 42$ & $7,24-30$ & & $\varnothing$ & $\varnothing$ & $\begin{array}{l}\pi \epsilon \rho \iota \alpha U \tau o v,-o v \\
-o \nu,-\omega\end{array}$ \\
\hline *43 & $7,31-37$ & & $\varnothing$ & $\varnothing$ & $\alpha u \tau \omega,-\mathrm{o \nu},-\mathrm{ou}$ \\
\hline *44 & $8,1-9$ & & $\varnothing$ & $\varnothing$ & $\alpha \cup \tau \omega,-\mathrm{ou},-\mathrm{ou}$ \\
\hline${ }^{*} 45$ & $8,10-13$ & & $\varnothing$ & $\varnothing$ & $\begin{array}{l}\alpha \cup \tau o s,-o v, \text { ouv } \\
-\omega, \pi \alpha \rho, \text { ou, } \\
-0 \nu\end{array}$ \\
\hline${ }^{* * *} 46^{198}$ & $8,14-21$ & $312 b, 26$ & o Inбous & $\varnothing$ & $\alpha \cup \tau \omega$ \\
\hline *47 & $8,22-26$ & & $\varnothing$ & $\varnothing$ & $\alpha \cup \tau \omega,-0 \nu$ \\
\hline **4 48 & $8,27-9,1$ & $313 b, 4$ & o Inбous & o Inбous & $\begin{array}{l}\alpha \cup \tau o v,-o v,-\omega, \\
-o \zeta,-\omega, \pi \epsilon \rho \iota- \\
\text { ov, }-\omega,-o v, \\
-o v\end{array}$ \\
\hline **4 49 & $9,2-13$ & $\begin{array}{l}314 b, 18 \\
348 b, 27 \\
314 b, 28 \\
315 b, 6\end{array}$ & 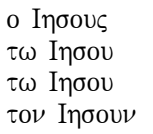 & 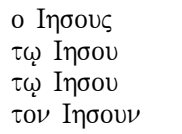 & 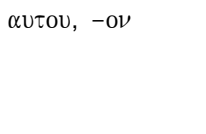 \\
\hline ***50 & $9,14-27$ & $\begin{array}{l}315 \mathrm{~b}, 28 \\
316 \mathrm{~b}, 17 \\
316 \mathrm{~b}, 21 \\
316 \mathrm{~b}, 30\end{array}$ & 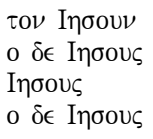 & 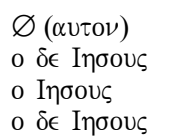 & 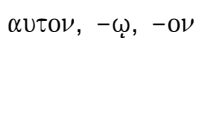 \\
\hline ****51 $51^{199}$ & $9,28-29$ & & $\varnothing$ & $\varnothing$ & $\alpha$ Uนov, $-\mathrm{ov},-\mathrm{ov}$ \\
\hline *52 & $9,30-32$ & & $\varnothing$ & $\varnothing$ & $\alpha$ Uนov, $-0 \nu$ \\
\hline *53 & $9,33-50$ & $317 b, 30$ & $\varnothing(\mathrm{o} \delta \epsilon)$ & 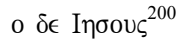 & $\alpha \cup \tau \omega$ \\
\hline ** 54 & $10,1-9$ & $319 b, 15$ & o Inбous & o $\delta \epsilon$ I $\eta \sigma o u \varsigma$ & $\begin{array}{l}\pi \rho 0 \varsigma \alpha \cup \tau o \nu,-0 \nu \\
-0 \nu\end{array}$ \\
\hline${ }^{* * *} 55^{201}$ & $10,10-12$ & & $\varnothing$ & $\varnothing$ & 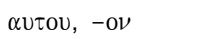 \\
\hline$* * 56$ & $10,13-16$ & $320 b, 3$ & o Inбous & 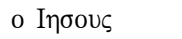 & $\alpha u \tau \omega,-o v$ \\
\hline ***57 & $10,17-22$ & $\begin{array}{l}320 \mathrm{~b}, 17 \\
320 \mathrm{~b}, 27\end{array}$ & 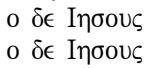 & 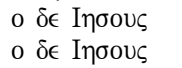 & $\begin{array}{l}\alpha \cup \tau o v,-0 \nu,-0 \nu, \\
-\omega\end{array}$ \\
\hline
\end{tabular}

194 Selon B03 cette séquence aurait dû être attribuée à la première rédaction.

195 Selon B03 cette séquence aurait dû être attribuée à la première rédaction.

196 Selon B03 cette séquence aurait dû être attribuée à la première rédaction.

197 Explication aux disciples à l'écart de la foule.

198 Explication aux disciples dans la barque à l'écart de la foule.

199 Explication aux disciples en particulier.

200 Selon B03 cette séquence ne pourrait être attribuée à la première rédaction.

201 Explication aux disciples au sein de la communauté à l'écart de la foule. 
Le Codex de Bèze : base indispensable pour une édition de Marc

\begin{tabular}{|c|c|c|c|c|c|}
\hline${ }^{* *} 58$ & $10,23-31$ & $\begin{array}{l}321 \mathrm{~b}, 2 \\
321 \mathrm{~b}, 10 \\
321 \mathrm{~b}, 16 \\
321 \mathrm{~b}, 21\end{array}$ & 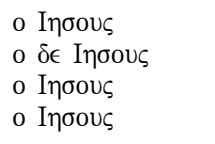 & 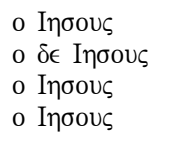 & $\begin{array}{l}\alpha u \tau o u,-o u,-o v, \\
-\omega\end{array}$ \\
\hline $\begin{array}{l}* * 59 \\
<^{* *} 60>\end{array}$ & $\begin{array}{l}10,32-34 \\
\text { ÉvS-1 }\end{array}$ & $322 b, 1$ & 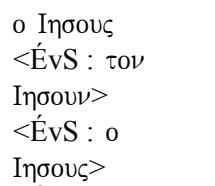 & 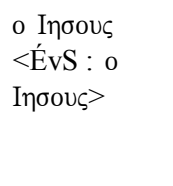 & $\begin{array}{l}\alpha u \tau \omega \\
<\alpha u \tau \omega,-\omega,-0 \nu, \\
-0 \nu>\end{array}$ \\
\hline$<* * 61>$ & ÉvS-2 & & 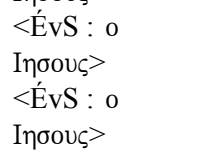 & & $\begin{array}{l}<\pi \rho \circ \varsigma \alpha U \tau O \nu, \sigma U \nu \\
-\omega>\end{array}$ \\
\hline${ }^{* *} 62$ & $\begin{array}{l}\mathrm{Mc} \\
10,35-45\end{array}$ & $\begin{array}{l}322 \mathrm{~b}, 22 \\
322 \mathrm{~b}, 26 \\
323 \mathrm{~b}, 1\end{array}$ & 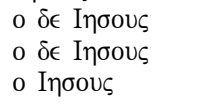 & 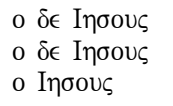 & $\alpha \cup \tau \omega,-\omega,-\omega$ \\
\hline$<^{* *} 63>$ & $\begin{array}{l}10,46 a+ \\
\text { ÉvS-3 }\end{array}$ & & 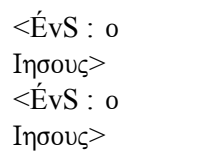 & & $<\alpha$ นโOU $>$ \\
\hline${ }^{* *} 64$ & $\begin{array}{l}10,46 \mathrm{~b}- \\
52\end{array}$ & $\begin{array}{l}323 \mathrm{~b}, 20 \\
323 \mathrm{~b}, 23 \\
323 \mathrm{~b}, 28 \\
323 \mathrm{~b}, 29 \\
323 \mathrm{~b}, 31\end{array}$ & 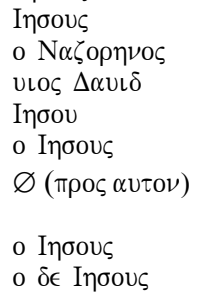 & 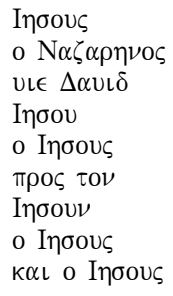 & $\begin{array}{l}\alpha \text { U } 0 v,-0 v, \pi \rho \circ \varsigma \\
-0 \nu,-\omega,-\omega\end{array}$ \\
\hline${ }^{* *} 65$ & $11,1-11$ & $\begin{array}{l}324 b, 12 \\
324 b, 18 \\
324 b, 20\end{array}$ & 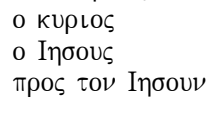 & 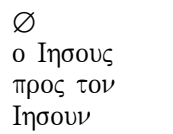 & $\alpha u \tau o u,-o u$ \\
\hline *66 & $11,12-14$ & & $\varnothing$ & $\varnothing$ & $\alpha u \tau o u$ \\
\hline $\begin{array}{l}* 67 \\
* * 68\end{array}$ & $11,15-19$ & $326 \mathrm{~b} 3$ & $\begin{array}{l}\varnothing \\
\text { o Inoous }\end{array}$ & $\begin{array}{l}\varnothing \\
\text { o Inoous }\end{array}$ & $\begin{array}{l}\alpha \cup \tau 0 \nu,-0 \nu,-0 \mathrm{U} \\
\alpha \cup \tau \omega\end{array}$ \\
\hline${ }^{* *} 69$ & $\begin{array}{l}11,27- \\
12,12+ \\
\text { Jn 7,53-8,1 }\end{array}$ & $\begin{array}{l}326 \mathrm{~b}, 26 \\
327 \mathrm{~b}, 7 \\
327 \mathrm{~b}, 8\end{array}$ & 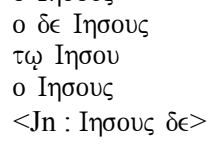 & 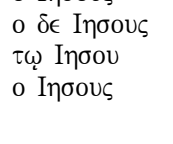 & $\begin{array}{l}\alpha \cup \tau o v, \pi \rho \circ-0 \nu, \\
-\omega\end{array}$ \\
\hline$<* * 70>$ & Jn $8,2-11$ & & 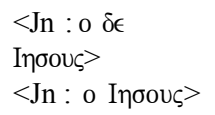 & & $\begin{array}{l}<\pi \rho \circ \varsigma \alpha U \tau O \nu,-\omega \\
-\mathrm{o \nu},-\mathrm{ov},-\omega>\end{array}$ \\
\hline${ }^{* *} 71$ & $\begin{array}{l}\text { Mc } \\
12,13-17\end{array}$ & $\begin{array}{l}328 \mathrm{~b}, 27 \\
328 \mathrm{~b}, 33\end{array}$ & 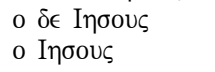 & 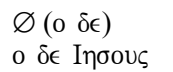 & $\begin{array}{l}\alpha u \tau O \nu,-0 \nu,-\omega, \\
\epsilon \pi^{\prime}-O \nu\end{array}$ \\
\hline${ }^{* *} 72$ & $\begin{array}{l}12,18-27 \\
12,28-34\end{array}$ & $\begin{array}{l}329 b, 22 \\
330 b, 7 \\
330 b, 26\end{array}$ & 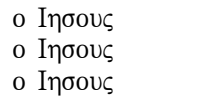 & 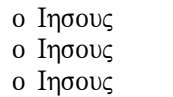 & $\begin{array}{l}\pi \rho 0 \varsigma \alpha \cup \tau o \nu,-0 \nu \\
\alpha \cup \tau \omega,-0 \nu,-\omega, \\
-0 \nu\end{array}$ \\
\hline
\end{tabular}




\begin{tabular}{|c|c|c|c|c|c|}
\hline **74 & $12,35-40$ & $330 \mathrm{~b}, 30$ & o Inoous & o Inoous & $\alpha \cup \tau o u$ \\
\hline **75 & $12,41-44$ & $331 b, 19$ & 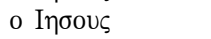 & $\varnothing^{202}$ & $\alpha$ $\alpha$ ข \\
\hline${ }^{* *} 76$ & $13,1-2$ & $332 b, 2$ & 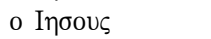 & 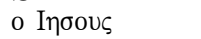 & $\alpha \cup \tau o v,-\omega,-o v$ \\
\hline **** $77^{203}$ & $13,3-36$ & $332 b, 15$ & 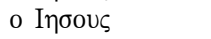 & o $\delta \epsilon$ Inбous & $\alpha u \tau o u,-0 \nu$ \\
\hline${ }^{*} 78$ & $14,1-2$ & & $\varnothing$ & $\varnothing$ & $\alpha \cup \tau o \nu$ \\
\hline$* * 79$ & $14,3-9$ & $\begin{array}{l}335 b, 27 \\
336 b, 5\end{array}$ & 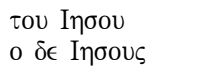 & 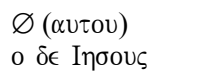 & $\alpha$ Uนov, $,-\mathrm{ov},-\mathrm{ov}$ \\
\hline *80 & $14,10-11$ & & $\varnothing$ & $\varnothing$ & $\alpha u \tau O \nu,-0 \nu$ \\
\hline *81 & 14,1216 & & $\varnothing$ & $\varnothing$ & $\alpha u \tau \omega,-\mathrm{OU},[-\mathrm{OU}]$ \\
\hline **82 82 & $14,17-21$ & $337 b, 14$ & 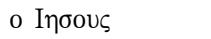 & 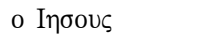 & $\alpha \cup \tau \omega$ \\
\hline *83 & $14,22-25$ & & $\varnothing$ & $\varnothing$ & \\
\hline **84 84 & $14,26-31$ & $\begin{array}{l}338 b, 7 \\
338 b, 16\end{array}$ & 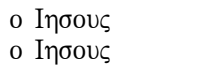 & 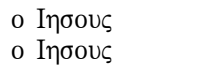 & $\alpha \cup \tau \omega$ \\
\hline *85 & $14,32-42$ & & $\varnothing$ & $\varnothing$ & $\begin{array}{l}\mu \in \tau^{\prime} \alpha \cup \tau o v, \alpha \pi^{\prime} \\
-o v,-\omega\end{array}$ \\
\hline *86 & $14,43-46$ & & $\varnothing$ & $\varnothing$ & $\begin{array}{l}\alpha \cup \tau o v,-0 \nu,-\omega, \\
-0 \nu,-\omega,-0 \nu\end{array}$ \\
\hline $\begin{array}{l}{ }^{* *} 87 \\
* 88\end{array}$ & $\begin{array}{l}14,47-50 \\
14,51-52\end{array}$ & $340 \mathrm{~b}, 7$ & $\begin{array}{l}\text { o } \delta \epsilon \text { I } \eta \sigma o u s \\
\varnothing\end{array}$ & $\begin{array}{l}\text { o Inбous } \\
\varnothing\end{array}$ & $\alpha \cup \tau o \nu$ \\
\hline **89 & $14,53-65$ & $\begin{array}{l}340 \mathrm{~b}, 17 \\
340 \mathrm{~b}, 27\end{array}$ & 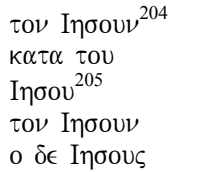 & 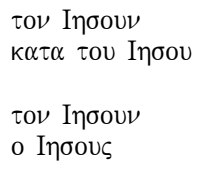 & $\begin{array}{l}\alpha \cup \tau \omega,-0 \nu, \kappa \alpha \tau, \\
-\mathrm{ov}, \kappa \alpha \tau,-\mathrm{ou} \\
-\omega,-\omega,-\mathrm{ov}, \\
-\mathrm{o \nu},-\omega,-0 \nu\end{array}$ \\
\hline **90 & $14,66-72$ & $342 b, 1$ & 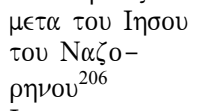 & 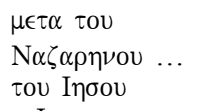 & \\
\hline${ }^{*} 91$ & $15,1-5$ & $\begin{array}{l}342 b, 17 \\
342 b, 22 \\
342 b, 32\end{array}$ & 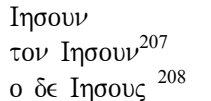 & 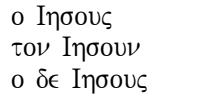 & $\alpha \mathrm{\tau} \tau \nu,-\mathrm{OU},-\mathrm{o \nu}$ \\
\hline *92 & $15,6-15$ & $343 b, 24$ & $\tau 0 \nu \delta \epsilon \operatorname{I\eta } \sigma o u \nu^{209}$ & $\tau o \nu$ I & $\alpha \cup \tau o \nu$ \\
\hline *93 & $15,16-20$ & & $\varnothing$ & $\varnothing$ & $\begin{array}{l}\alpha u \tau o \nu,-0 \nu,-\omega, \\
-0 \nu,-0 \nu,-\omega, \\
-0 \nu,-0 \nu,-0 \nu\end{array}$ \\
\hline *94 & $15,21-32$ & & $\varnothing$ & $\varnothing$ & $\begin{array}{l}\alpha u \tau o v,-0 \nu,-\omega, \\
-0 \nu,-0 u,-0 \nu, \\
-o v, \sigma \cup \nu,-\omega,\end{array}$ \\
\hline
\end{tabular}

202 Selon B03 cette séquence aurait dû être attribuée à la première rédaction.

203 Explication à Pierre, Jacques, Jean et André en particulier.

${ }^{204}$ Mention du nom indispensable, car cette séquence vient actuellement après la séquence dont le seul protagoniste était le jeune homme.

${ }^{205}$ Mention du nom indispensable, car Pierre a été mentionné dans le v. précédent.

206 Discours direct.

${ }^{207}$ Mention du nom indispensable, car en première rédaction cette séquence venait immédiatement après la ${ }^{*}$ Séq. 88 dont le seul protagoniste était le jeune homme.

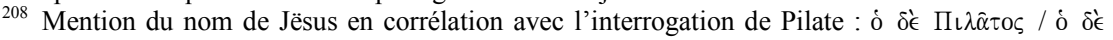
'Iฤбoûs.

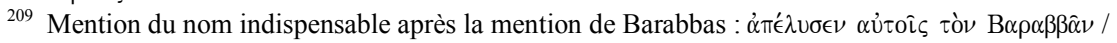

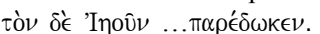




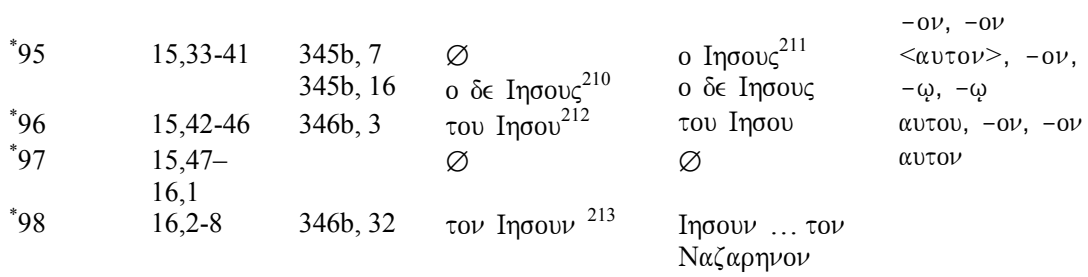

Le nombre total de fréquences du nom de Jésus dans le Codex de Bèze s'élève à $112 \times(86 \times$ seulement dans le Codex Vaticanus $)$. Les deux codexs ont en commun 40 séquences où l'absence du nom est total ; ont doit ajouter 2 séquences au compte de D05 et 7 séquences au compte de B03. C'est-à-dire que l'absence ou la présence du nom de Jésus diffèrent de façon remarqueable de l'un à l'autre codex. Cette constatation peut être décisive à l'heure d'assigner une séquence à la première ou à la deuxième rédaction.

En le regardant de plus près, je me suis aperçu que ce phénomène n'apparaissait pas seulement dans des séquences isolées, mais qu'il y avait de séries de séquences où figure constamment le nom de Jésus, ${ }^{214}$ et d'autres séries où il n'est jamais mentionné ou bien il est remplacé par le pronom. ${ }^{215}$ Ces différences seraient-elles dûes à des sources diverses, ou plutôt seraient-elles le

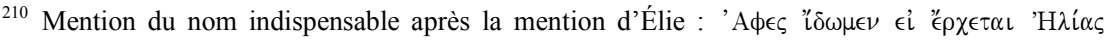

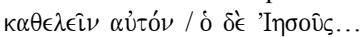

211 Selon $\mathrm{B} 03$ cette séquence ne pourrait être attribuée à la première rédaction.

${ }^{212}$ Mention du nom indispensable, a fin de préciser que la demande de Joseph d'Arimathie visait «le cadavre de Jésus», puisqu'il y avait d'autres qui avaient été crucifiés avec lui.

213 Discours direct.

214 P.ex., Séqs. 25-31 (5,1-6,6a) : le démoniaque gérasénien, l'hémorroïsse et la mort de la petite fille d'un des chefs de synagogue ; Séqs. 35-39 : première multiplication des pains dans un lieu désert, promenade de Jésus sur la mer et guérisons au pays de Gennésareth (6,30-56); Séqs. 48-50 : première annonce de la Passion, Transfiguration et expulsion de l'esprit sourd-muet $(9,2-27)$; Séqs. 54-65: thème du chemin qui monte à Jérusalem en partant de « la région de la Judée (la Pérée) au delà du Jourdain », traversée du Jourdain, passage par Jéricho, guérison de l'aveugle assis au bord du chemin de Jésus et entrée messianique à Jérusalem (10,1-11,11. Les trois séquences récupérées de l'Évangile Secret de Marc [Séqs. 60-61, 63] faisaient partie à l'origine de cette série); Séqs. 68-77 : dispute au Temple avec les divers représentants du pouvoir religieux, politique et intellectuel (11,20-13,37. La séquence de la femme adultère [Séq. 70] faisait partie à l'origine de cette série).

215 P.ex., Séqs. 6-10 : enseignement à la synagogue de Capharnaüm, guérisons dans la maison de Simon et André et devant la porte, retraite dans un lieu solitaire et parcours de toute la Galilée (1,21-45) ; Séqs. 17-19 et 21-24 : réactions diverses à l'institution des Douze, enseignement en paraboles sur le Royaume de Dieu au bord de la mer, apaisement de la tempête (3,7b-21 et $3,31-4,41)$; Séqs. 40-45: discussion sus les traditions pharisiennes, retraite de Jésus à l'étranger (Tyr, Sidon, la Décapole), guérison d'un sourd-muet, deuxième multiplication des pains dans un lieu désert et retour en barque à la région de Mélégada où les Pharisiens lui demandaient le signe venant du ciel $(7,1-8,13)$. 
résultat de deux rédactions entreprises par Marc lui-même à des époques différentes et dans des situations très diverses?

\subsection{Séquences dédoublées}

Un deuxième phénomène m'a permis d'avancer vers une réponse à ma question initiale : dans le cas de séquences doublées (par exemple, deux appels de disciples, deux multiplications des pains, deux tempêtes, deux aveugles, deux sourds-muets, deux procès instruits contre Jésus, religieux, l'un, et politique, l'autre), dans l'une est mentionné toujours le nom de Jésus et dans l'autre on fait une référence à lui moyennant le pronom ou simplement avec le verbe en troisième personne du singulier quand c'est lui qui porte l'initiative.

Après les avoir examinés à fond, autant les modèles comme les doublets, je suis arrivé à la conclusion que les séquences où il n'y a aucune référence à Jésus étaient plus primitives que les autres. L'utilisation exclusive d'un verbe personnel, du pronom ou de l'article pronominal de la part du rédacteur Marc dans presque la moitié des séquences serait un indice de la proximité de son auditoire dans l'espace et le temps à la personne de Jésus. Marc aurait utilisé ces séquences $\left(^{*}\right)$ comme modèle pour créer plus tard des doublets $\left(^{* *}\right)$, en deuxième rédaction.

Ainsi, j'ai pu séparer deux niveaux de rédaction, dont la rédaction la plus ancienne est bien plus courte que l'évangile dans son état actuel (moins que la moitié) mais qui possède sa propre cohérence interne. Voyons quelques exemples.

Sur le modèle de l'appel de Jacques (Lévi, B03), fils d'Alphée ('Séq. 12. 2,13-14), appartenant à la première rédaction, Marc aurait anticipé en deuxième rédaction un doublet en forme de diptyque, l'appel de Simon et André, premièrement, et celui de Jacques et Jean, à la suite ( ${ }^{* *}$ Séq. 5. 1,16-20).

Prenant comme modèle la tempête apaisée ('Séq. 24. 4,35-41), Marc aurait rédigé une deuxième séquence où Jésus se promène sur les eaux pendant que la barque était au milieu de la mer et les disciples s'épuisaient à ramer car le vent leur était contraire $\left({ }^{* *}\right.$ Séq. 38. 6,47-52).

A partir du modèle de la multiplication de sept pains dans un lieu isolé ('Séq. 44. 8,1-9), en territoire de la Décapole (cf. 7,31), Marc aurait composée une seconde multiplication de cinc pains ( ${ }^{* *}$ Séq. 37. 6,35-46: cette multiplication se trouve maintenant en premier lieu), mais cette fois-ci en territoire juif (cf. 6,45), toujours dans un lieu désert. On peut vérifier dans la Table 4 cidessus que, selon D05 uniquement, dans la séquence dedoublée s'explicite trois fois le nom de Jésus (vv. 36, 38, 39: aucune des trois vll n'ont pas été enregistrées dans Nestle-Aland), et non pas dans la séquence modèle. Si j'avais 
voulu controler mon hypothèse en partant du texte alexandrin, je me serais trouvé dans une impasse, puisque j'aurais dû assigner toutes les deux multiplications à la première rédaction, un obstacle impossible de surmonter qui aurait fait non-viable mon point de départ. Je donne en caractères grasses les $v l l$ divergentes parmi D05 et B03 ; soulignées, les vll qui se trouvent exclusivement dans D05, et en italiques quan il y a un changement d'ordre des mots par rapport avec B03; le symbole $\uparrow$ indique qu'il y a un plus dans B03. Voici en parallèle le texte de la séquence modèle ( ${ }^{*}$ Séq. 44$)$ et celui de son dédoublement ( ${ }^{* *}$ Séq. 37$)$ suivant le Codex de Bèze.

\section{Table 4}

Multiplication des sept pains (modèle) et des cinc pains (dédoublement) selon D05

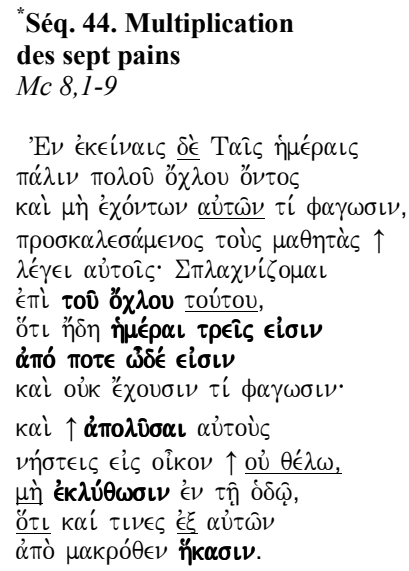

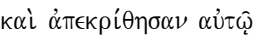

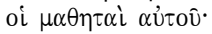

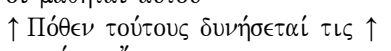

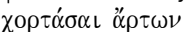

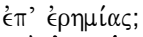

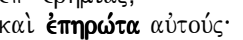

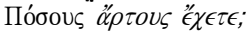

oi $\delta^{\prime} \epsilon \in \hat{\imath} \pi \nu^{\prime}$.

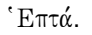

\author{
** Séq. 37. Multiplication \\ des cinc pains \\ Mc 6,35-46

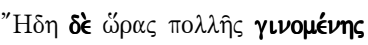

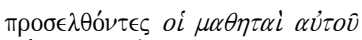 \\ $\lambda \dot{\boldsymbol{\epsilon}} \gamma o v \sigma \iota \nu \alpha \dot{v} \tau \hat{\omega}$

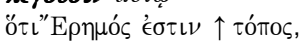

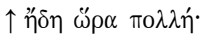

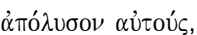 \\ ¿ $\nu \alpha \alpha \dot{\alpha} \pi \lambda \theta$ ó $\nu \tau \epsilon \zeta$

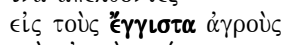

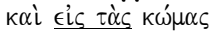

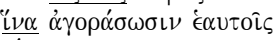

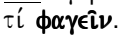

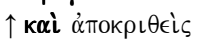

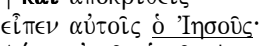

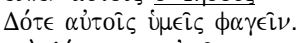

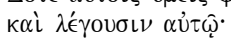

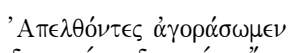

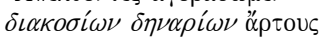

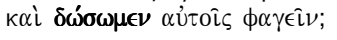

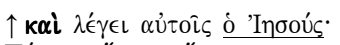

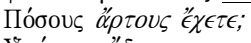 \\ $Y \pi \alpha ́ \gamma \epsilon \tau \epsilon$, ’ $\delta \in \tau \epsilon$.

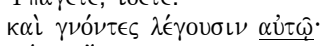

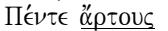

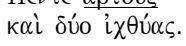




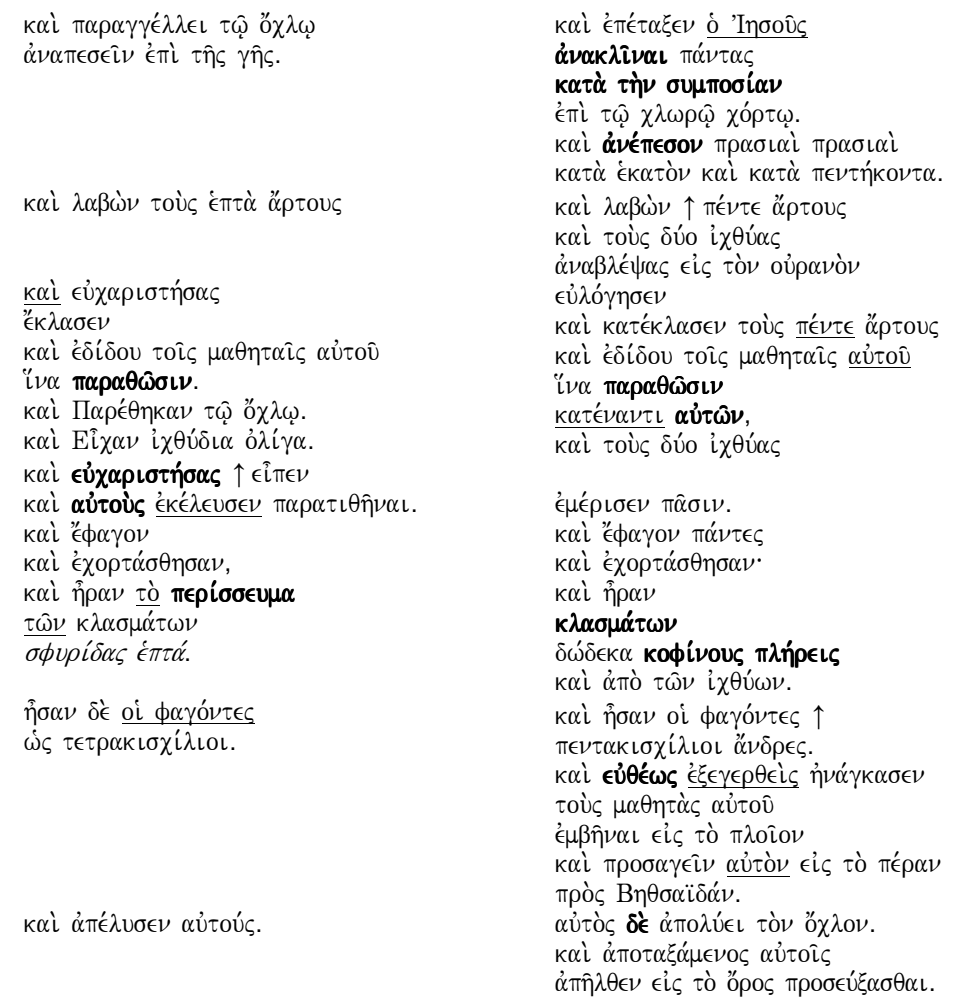

À deux reprises Marc fait référence à des aveugles. La première fois, à Béthanie au delà du Jordain ${ }^{216}$ (D05 [à Bethsaïde, B03]), on lui amène un aveugle ("Séq. 47. 8,22-26: séquence modèle); la deuxième fois, à la sortie de Jéricho, c'est le fils de Timée (Précieux, D05 [Bartimée, B03]), un aveugle qui était assis au bord du chemin en mendiant ( ${ }^{* *}$ Séq. $64.10,46 b-52$ : doublet de deuxième rédaction), qui se mit à crier.

De nouveau, Marc fait référence à des sourds-muets à deux endroits. La première fois, en plein territoire de la Décapole, on amène à Jésus un sourd qui parlait avec difficulté ("Séq. 43. 7,31-37 : séquence modèle) ; la deuxième fois, quand on descendait de la montagne de la Transfiguration, le père d'un enfant qui avait un esprit muet et sourd et qui auparavant avait demandé aux disciples de l'expulser, supplie Jésus de venir en aide à son incrédulité ( ${ }^{* * *}$ Séq. 50. 9,14-

${ }^{216}$ Lieu où Jean baptisait, selon l'évangéliste Jean (Jn 1,28). 
27 : séquence dédoublée). Il y a des parallèles verbaux, mais ils sont surtout d'ordre structurel. Le texte du doublet est beaucoup plus long et détaillé.

3.3 Questions posées par les disciples, dans un lieu séparé de la foule, sur des thèmes déjà traités auparavant

Un troisième phénomène m'a suggéré la possibilité d'un troisième niveau de rédaction : il y a six séquences $\left({ }^{* * *}\right)$ où Jésus répond à des questions posées par ses disciples, dans un lieu séparé de la foule, sur des thèmes dont il avait déjà parlé auparavant :

1. ${ }^{* * *}$ Séq. 23 (Mc 4,10-25.34). Après la Parabole du semeur, annoncée par Jésus à la foule au bord de la mer ('Séq. 22A [4,1-9]), se trouve l'explication de cette parabole adressée maintenant «aux disciples » («à ceux de son entou-rage avec les Douze », B03), dans un lieu à part «Quand il fut à part de la foule » $(4,10)$. Par la suite, Jésus continue l'enseignement adressé à la foule, sans autre indication ( Et il [leur] disait », 4,26) en leur proposant deux autres paraboles ("Séq. 22B [4,26-32]) : la Parabole du grain qui pousse tout seul (4,26-29) et la Parabole du grain de sénevé (4,30-32), comme Marc avait anticipé au debut : « Il leur ensegnait

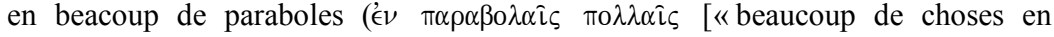

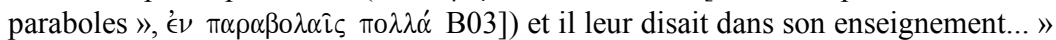
$(4,2)$. En guise de conclusion aux paraboles, Marc commente : «C'est par un grand

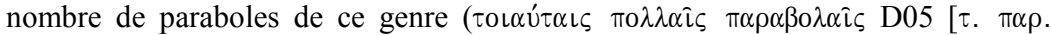

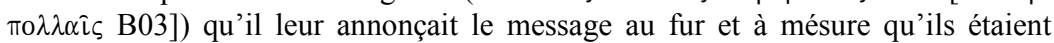
capables de l'entendre » $\left({ }^{*} 4,33\right)$. En troisième rédaction il ajoutera encore : «Et il

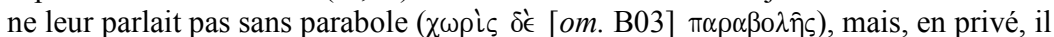
expliquait tout à ses disciples (+ « eux-mêmes », B03)» $\left({ }^{* * *} 4,34\right)$.

2. ${ }^{* * *}$ Séq. 41 (Mc 7,17-23) Après l'enseignement sur le pur et l'impure (7,14-16) Marc attache l'explication de la parabole: "Quand il fut rentré dans la commu-

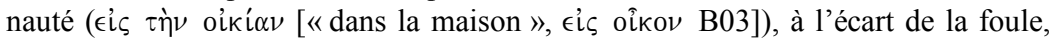
ses disciples l'interrogèrent sur la parabole $»(7,17)$.

3. ${ }^{* * *}$ Séq. 46 (Mc 8,14-21) Mais ce qui m'a convaincu de l'existence de ce troisième niveau a été la récapitulation des deux multiplications des pains dans Mc 8,14-21 que j'avais déjà attribuées (suivant les critères élaborés ci-dessus) l'une, à la première rédaction (*Séq. 44$)$ et l'autre, à la deuxième $\left({ }^{* *}\right.$ Séq. 37$)$. Voici leur récapitulation dans la ${ }^{* * *}$ Séq. 46.

Table 5

Récapitulation des deux multiplications des pains

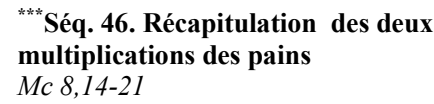




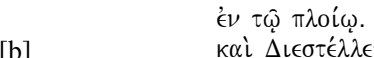

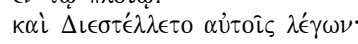

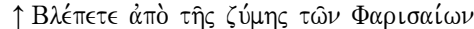

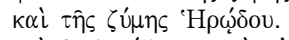

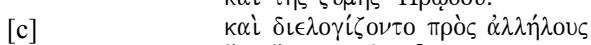

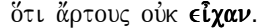

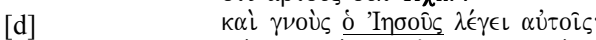

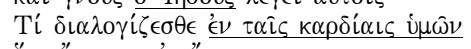

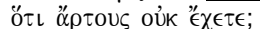

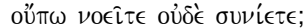

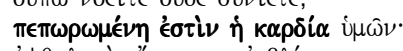

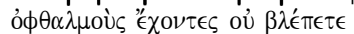

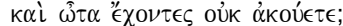

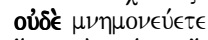

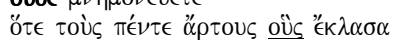

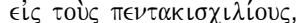

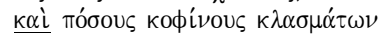

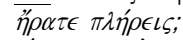

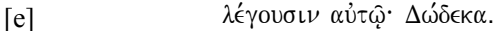

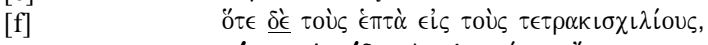

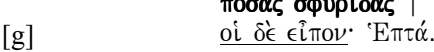

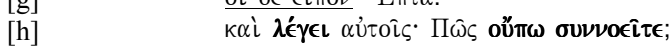

«Les disciples (om. B03) avaient oublié de prendre des pains; ( « et», B03) ils n'avaient qu'un seul pain avec eux dans la barque ... et ils se mirent à faire

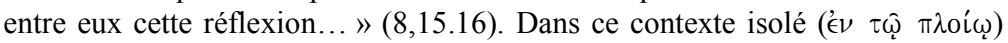
Jésus s'en aperçut et leur dit: "Pourquoi vous faites cette reflexion dans vos coeurs (om. B03) que vous n'avez pas de pain? Vous ne comprenez pas encore et vous ne saisissez pas? Votre coeur il est émoussé! ( « Avez vous votre coeur bouché? », B03). Ayant des yeux vous ne voyez pas et ayant des oreilles vous n'entendez pas? Ne («Et ne », B03) vous rappelez-vous pas, quand j'ai rompu les cinc pains, pour les cinc mil hommes, combien de corbeilles pleines de morceaux vous avez ramassées ? " Ils lui répondent : "Douze. » Nous avons ici une très claire référence à la première multiplication des pains que, selon les critères énoncés ci-dessus, j'ai considérée qu'elle appartenait à la deuxième rédaction $\left({ }^{* *}\right.$ Séq. $37[\mathrm{Mc} 6,35-44)$. Jésus continue en leur disant : «Et quand j'ai rompu les sept pains pour les quatre mil, combien de paniers (+ «pleins » B03) de morceaux avez-vous ramassées ? » Et ils lui disent : «Sept.» Cette fois-ci, il fait référence à la seconde multiplication des pains, que par les mêmes critères j'avais assignée à la première rédaction ( ${ }^{*}$ Séq. 44 [Mc 8,1-10). Jésus conclut : "Alors il leur dit (« disait» B03) : " Comment ne comprenezvous pas encore ? (“Comment n'entendez-vous rien ? ”, B03) ”» En troisième rédaction, donc, Marc souligne une fois de plus que les disciples n'ont rien compris.

La manière dont Marc a fait référence ici au double enseignement qu'accordait Jésus à ses disciples sur les pains fonctionne comme une espèce de « pierre de Rosetti » qui permet de séparer les trois niveaux de rédaction. Il 
est bien possible que les ordres précis donnés par Jésus aux disciples : «Gardez-vous du levain des Pharisiens et du levain d'Hérode », en distingueant intentionnellement un levain de l'autre, correspondent respectivement aux deux multiplications qu'il va leur rappeler par la suite : avec la première multiplication (suivant l'ordre actuel de l'évangile) Jesús aurait donné le signe messianique par excellence dans « un lieu désert », très prôche des villes vers lesquelles une grande foule avait accouru, à pied (6,32-33), c'est-à-dire en territoire juif mais à l'écart des Pharisiens qui y exerçaient leur influence ; avec la seconde multiplication (de première rédaction) il aurait donné le signe messianique dans « un lieu solitaire » $(8,4)$, « en plein territoire de la Décapole » $(7,31)$, c'est-à-dire en territoire païen où le roi juif Hérode n'avait aucune jurisdiction.

Il y a encore trois autres séquences où on retrouve le même procédé :

4. ${ }^{* * *}$ Séq. 51 (Mc 9,28-29) : «Quand il fut rentré à la maison, ses disciples lui demandèrent en privé : “Pourquoi nous n'avons-nous pu l'expulser nous-mêmes ? "»"

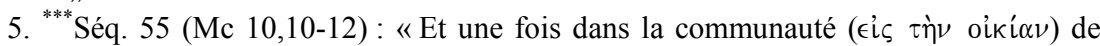
nouveau ses («les », B03) disciples l'interrogèrent sur cette parole (« ce point», B03). »

6. ${ }^{* * *}$ Séq. 77 (Mc 13,3-37) : «Et comme il était assis sur le mont des Oliviers en face du Temple, Pierre, Jacques, Jean et André l'interrogèrent (« [Pierre] l'interrogea », B03) à part : " Dis-nous quand cela aura lieu " et "Quel sera le signe que tout cela va s'accomplir?"»(13,3-4).

\section{En guise de conclusion}

J'ai voulu présenter par l'analyse des séquences selon le Codex de Bèze la justification du choix de ce manuscrit pour le texte de base pour une édition de l'Évangile de Marc. Il n'y a que ce manuscrit grec qui laisse encore ces traces de l'œuvre rédactionnelle de l'auteur. Lorsque l'on examine chaque niveau de rédaction indépendamment, on constate que le premier a un fil très cohérent, le deuxième s'insère dans le premier afin d'en développer certains aspects non traités dans le premier, et le troisième présente un enseignement destiné uniquement aux disciples seuls. L'étude des différences théologiques entre les différents niveaux est fort intéressante, et est d'une grande valeur pour l'étude des variantes textuelles lorsqu'elles sont considérées dans le contexte de l'histoire de l'Église. C'est une question que j'examine en détail dans les notes qui accompagneront l'édition du texte, à laquelle je réfère mes lecteurs.

Recibido / Received: 3/05/2007

Aceptado / Accepted: 18/12/ 2007 